TRANSACTIONS OF THE

AMERICAN MATHEMATICAL SOCIETY

Volume 356, Number 1, Pages 291-317

S 0002-9947(03)03361-0

Article electronically published on August 25, 2003

\title{
ANALYSING FINITE LOCALLY $s$-ARC TRANSITIVE GRAPHS
}

\author{
MICHAEL GIUDICI, CAI HENG LI, AND CHERYL E. PRAEGER
}

\begin{abstract}
We present a new approach to analysing finite graphs which admit a vertex intransitive group of automorphisms $G$ and are either locally $(G, s)$ arc transitive for $s \geq 2$ or $G$-locally primitive. Such graphs are bipartite with the two parts of the bipartition being the orbits of $G$. Given a normal subgroup $N$ which is intransitive on both parts of the bipartition, we show that taking quotients with respect to the orbits of $N$ preserves both local primitivity and local $s$-arc transitivity and leads us to study graphs where $G$ acts faithfully on both orbits and quasiprimitively on at least one. We determine the possible quasiprimitive types for $G$ in these two cases and give new constructions of examples for each possible type. The analysis raises several open problems which are discussed in the final section.
\end{abstract}

\section{INTRODUCTION}

Let $\Gamma$ be a connected graph with vertex set $V \Gamma$, edge set $E \Gamma$ and adjacency denoted by $\sim$. An $s$-arc in $\Gamma$ is an $(s+1)$-tuple $\left(v_{0}, v_{1}, \ldots, v_{s}\right)$ of vertices in $\Gamma$ such that $v_{i} \sim v_{i-1}$ and $v_{j-1} \neq v_{j+1}$ for each $i=1, \ldots, s$ and $j=1, \ldots, s-1$. Given $G \leqslant \operatorname{Aut}(\Gamma)$ we call $\Gamma$ locally $(G, s)$-arc transitive if $\Gamma$ contains an $s$-arc and given any two $s$-arcs $\alpha$ and $\beta$ starting at the same vertex $v$, there exists an element $g \in G_{v}$ mapping $\alpha$ to $\beta$. We say $\Gamma$ is locally $s$-arc transitive if it is locally $(G, s)$-arc transitive for some $G \leqslant \operatorname{Aut}(\Gamma)$. If all vertices in $\Gamma$ have valency at least two, local $(G, s)$-arc transitivity implies local $(G, s-1)$-arc transitivity. We concentrate on the case where $s \geq 2$ as local 1-arc transitivity is equivalent to edge transitivity, a far more general class of graphs.

Locally $s$-arc transitive graphs are intimately linked to many areas of mathematics. A locally $s$-arc transitive graph of girth $g$ with at least one vertex of valency greater than two satisfies $s \leq(g+2) / 2$. (The girth of $\Gamma$ is the smallest integer $g$ such that $\Gamma$ contains a cycle of length $g$.) When equality holds, various interrelated objects arise such as generalised $(s-1)$-gons, groups with a $(B, N)$-pair of rank two, Moufang polygons and Tutte's $m$-cages. These connections are explained in more detail in [33. Locally $s$-arc transitive graphs also arose in the work of Delgado and Stellmacher in [6] on weak $(B, N)$-pairs of rank two. Other interesting examples arise naturally from incidence graphs of various structures, for example the points and lines of an affine space (Example 4.2) or the vertices and maximal cliques of a Hamming graph (Example 4.3).

Received by the editors November 22, 2002.

2000 Mathematics Subject Classification. Primary 05C25, $20 \mathrm{~B} 25$.

This paper forms part of an Australian Research Council large grant project which supported the first author. The second author was supported by an ARC Fellowship. 
A group $G$ acting on a set $\Omega$ is said to be primitive if the only partitions of $\Omega$ that $G$ preserves are the trivial ones, that is, the whole set or the partition into sets of size one. We call a graph $G$-locally primitive if for each vertex $v$, the stabiliser $G_{v}$ acts primitively on $\Gamma(v)$, where $\Gamma(v)$ is the set of vertices adjacent to $v$. We say $\Gamma$ is locally primitive if it is $G$-locally primitive for some automorphism group $G$. Now suppose that each vertex of a graph $\Gamma$ has valency at least two. Then $\Gamma$ is locally $(G, 2)$-arc transitive if and only if for every vertex $v, G_{v}$ acts 2 -transitively on the set $\Gamma(v)$ (see Lemma 3.2). Thus locally $(G, 2)$-arc transitive graphs are $G$-locally primitive.

Let $\Gamma$ be a locally $(G, s)$-arc transitive graph. If $G$ is transitive on vertices, then $G$ acts transitively on the set of $s$-arcs of $\Gamma$, that is, $\Gamma$ is $(G, s)$-arc transitive. Interest in such graphs goes back to Tutte's seminal work ([29], 30]) showing that $(G, s)-\operatorname{arc}$ transitive graphs of valency three satisfy $s \leq 5$. Later, Weiss 32 proved that if the valency is at least three, then $s \leq 7$. Unlike Tutte's ingenious elementary methods, Weiss's proof uses the classification of finite 2-transitive groups and thereby the finite simple groups classification. Note that cycles are $s$-arc transitive for all $s$. The second author [19] has determined all $(G, s)$-arc transitive graphs with $s \geq 4$ for which $G$ acts primitively or biprimitively on vertices. This result and others mentioned below also depend on the finite simple group classification.

If $G$ does not act transitively on vertices and all vertices have valency at least two, then as $G$ is edge transitive, $\Gamma$ is bipartite and $G$ has two orbits on vertices: the two parts of the bipartition. Weiss (see 31]), has proved that $s \leq 7$ in the valency three case while Stellmacher announced in Siena in 1996 that $s \leq 9$ for locally $(G, s)$-arc transitive graphs with all vertices of valency at least three (see [27]). The maximum $s=9$ is attained by the classical generalised octagons. We see in Corollary [3.13 that if $\Gamma$ has a vertex of valency two and a vertex of valency at least three, then $s \leq 13$. If all vertices have valency two, then the graph is a cycle. Most work on locally $(G, s)$-arc transitive graphs has centered around the local structure and more specifically the possible point stabilisers of two adjacent vertices. The theory of amalgams plays a crucial role in such a strategy and Goldschmidt's 14 paper determines all possible vertex stabilisers in the valency three case. Stellmacher and van Bon are currently attempting to determine all possible vertex stabilisers for locally $(G, 4)$-arc transitive graphs where all vertices have valency at least three.

Studying certain quotients of graphs has been very successful in investigating various families of graphs, for example distance transitive graphs [26] and $s-\operatorname{arc}$ transitive graphs [23]. We now outline this method for the latter case. Let $\Gamma$ be a graph with a group of automorphisms $G$. If $G$ has a normal subgroup $N$ which acts intransitively on vertices, then we define the quotient graph $\Gamma_{N}$ to have vertex set the $N$-orbits on $V \Gamma$, and two $N$-orbits $B_{1}$ and $B_{2}$ are adjacent in $\Gamma_{N}$ if and only if there exist $v \in B_{1}$ and $w \in B_{2}$ such that $v$ and $w$ are adjacent in $\Gamma$. The original graph $\Gamma$ is said to be a cover of $\Gamma_{N}$ if $\left|\Gamma(v) \cap B_{2}\right|=1$ for each edge $\left\{B_{1}, B_{2}\right\}$ in $\Gamma_{N}$ and $v \in B_{1}$. If $\Gamma$ is a nonbipartite $(G, s)$-arc transitive graph, then $\Gamma$ is a cover of $\Gamma_{N}$ and $\Gamma_{N}$ is a $(G / N, s)$-arc transitive graph. This reduces the problem to finding all examples where $G$ acts quasiprimitively on vertices and then studying their covers. (A transitive permutation group $G$ on a set $\Omega$ is said to be quasiprimitive if every nontrivial normal subgroup of $G$ acts transitively on $\Omega$.) There are eight types of quasiprimitive groups (these are described in Section 21), and it was shown 
in [23] that only four of them, HA, TW, AS and PA, occur for nonbipartite $(G, s)-$ arc transitive graphs. All graphs arising in the HA case were determined in [16], while Baddeley [1] provided a nice characterisation for the graphs of type TW. A similar reduction for the vertex transitive, bipartite case was done in [22].

The purpose of this paper is to initiate a similar program for locally $(G, s)$ arc transitive graphs for which $G$ acts intransitively on vertices. We see that the appropriate quotients to take are those with respect to the orbits of a certain normal subgroup which is intransitive on both the $G$-orbits. The O'Nan-Scott Theorem for quasiprimitive groups is then used to study the "basic" graphs, those for which there are no suitable normal quotients. Wherever possible we will prove our results in the more general case of $G$-vertex intransitive, $G$-locally primitive graphs. Note that if $\Gamma$ is a bipartite $(G, s)$-arc transitive graph, then $\Gamma$ is locally $\left(G^{+}, s\right)$-arc transitive for the index two subgroup $G^{+}$of $G$ which fixes both parts of the bipartition. Our results applied to $G^{+}$provide a slightly different analysis for bipartite $(G, s)$-arc transitive graphs to the one in [22].

Our first result is a reduction theorem, but before stating it we need the following definition. A graph $\Gamma$ is called a star if there exists a vertex $v$ adjacent to every other vertex of $\Gamma$ and all vertices apart from $v$ have valency one, that is, $\Gamma=K_{1, n}$ for some positive integer $n$. We call the vertex $v$ the central vertex of $\Gamma$.

Theorem 1.1. Let $\Gamma$ be a finite connected $G$-locally primitive graph such that $G$ has two orbits $\Delta_{1}$ and $\Delta_{2}$ on the set of vertices of $\Gamma$ with each $\left|\Delta_{i}\right|>1$. Let $N$ be a normal subgroup of $G$, the maximal subject to being intransitive on both orbits $\Delta_{1}$ and $\Delta_{2}$. Then $\Gamma_{N}$ is a $(G / N)$-locally primitive graph, $\Gamma$ is a cover of $\Gamma_{N}$ and $\Gamma_{N}$ satisfies at least one of the following:

(1) $\Gamma_{N} \cong K_{n, n^{\prime}}$.

(2) $G / N$ acts faithfully and quasiprimitively on both of its orbits on $V \Gamma_{N}$.

(3) $G / N$ acts faithfully on both of its orbits on $V \Gamma_{N}$ but only acts quasiprimitively on one of them. Furthermore, there exists $M \triangleleft G$ containing $N$, transitive on one $G$-orbit but intransitive on the other, and $\Gamma_{M}$ is a star.

Moreover, if $\Gamma$ is locally $(G, s)$-arc transitive, then $\Gamma_{N}$ is locally $(G / N, s)$-arc transitive. In fact, every star occurs as a normal quotient of some locally 3-arc transitive graph.

This suggests that to investigate locally $(G, s)$-arc transitive graphs we should concentrate on three problems: determining such graphs where $G$ acts quasiprimitively on both of its orbits, determining such graphs where $G$ acts quasiprimitively on only one of its orbits, and investigating locally $s$-arc transitive covers of these two types of graphs and of complete bipartite graphs. A similar result for the case where $\Gamma$ is locally $s$-arc transitive for $s \geq 4$ and valency at least three was obtained in [17], but the situation is different there, as the stars and complete bipartite graphs do not occur. See also Lemma 5.6.

Now $\operatorname{Aut}(\Gamma)$ may or may not act transitively on the vertices of $\Gamma$. Hence $\Gamma$ is not necessarily regular and $G$ may act differently on each of its orbits. We say that $G$ is of type $\{X, Y\}$ if $G$ acts quasiprimitively of type $X$ on one $G$-orbit and quasiprimitively of type $Y$ on the other $G$-orbit, where the types $X$ and $Y$ refer to quasiprimitive types as described in Section 2 Often when $X=Y$, we will relax notation and say that $G$ is of type $X$. The following theorem determines the 
possibilities for the type of $G$ and shows that the two actions of $G$ are usually of the same type.

Theorem 1.2. Let $\Gamma$ be a finite $G$-locally primitive connected graph such that $G$ has two orbits on vertices and $G$ acts faithfully and quasiprimitively on both orbits with type $\{X, Y\}$. Then either $X=Y$, or $\{X, Y\}=\{\mathrm{SD}, \mathrm{PA}\}$ or $\{\mathrm{CD}, \mathrm{PA}\}$, and examples exist in each case. Furthermore, if $\Gamma$ is locally $(G, s)$-arc transitive with $s \geq 2$, then either $X=Y \in\{\mathrm{HA}, \mathrm{TW}, \mathrm{AS}, \mathrm{PA}\}$ or $\{X, Y\}=\{\mathrm{SD}, \mathrm{PA}\}$, and again examples exist in each case.

Examples of locally $(G, s)$-arc transitive graphs where $G$ is quasiprimitive of type HA, TW, AS and PA will be provided in Lemma 3.3 while Example 4.1 gives examples where $G$ is of type $\{\mathrm{SD}, \mathrm{PA}\}$. Examples of $G$-locally primitive graphs of the remaining possible types can be found in Example 6.6. Note that Theorem 1.2 corresponds closely to the result in [23] that for a $(G, s)$-arc transitive, nonbipartite graph where $G$ acts on vertices quasiprimitively, $G$ has quasiprimitive type HA, TW, $\mathrm{AS}$ or PA [23]. If $G$ is of quasiprimitive type HA on both of its orbits, $\Gamma$ was shown to be vertex transitive in [15, Lemma 3.2] and this situation is discussed in [16]. When $G$ acts quasiprimitively on only one orbit we have the following theorem.

Theorem 1.3. Let $\Gamma$ be a finite locally $(G, s)$-arc transitive connected graph with $s \geq 2$ such that $G$ acts faithfully on both of its orbits $\Delta_{1}$ and $\Delta_{2}$ but only acts quasiprimitively on $\Delta_{1}$. Then the quasiprimitive action of $G$ on $\Delta_{1}$ is of type HA, $\mathrm{HS}, \mathrm{AS}, \mathrm{PA}$ or TW, and examples exist for each of these types.

We provide examples for each of the five possibilities in Section 4. For the more general class of $G$-locally primitive graphs, the quasiprimitive action on $\Delta_{1}$ may be of the three remaining types as seen in Example 7.2

In Section 3, we give some general constructions of edge transitive graphs and in particular we characterise locally $s$-arc transitive graphs having a vertex of valency two and show that for such graphs $s \leq 13$ (see Theorem 3.10] and Corollary 3.13). We provide examples in Section 4 verifying our claims about the existence of locally $(G, s)$-arc transitive graphs with $G$ quasiprimitive of various types. Section [5] is devoted to the proof of Theorem [1.1, Section [6] to the proof of Theorem [1.2] and Section 7 for Theorem 1.3 . Finally in Section 8 we discuss some open problems and future directions arising from this work.

\section{QUASIPRIMITIVE GROUPS}

Recall that a transitive permutation group $G$ on a set $\Omega$ is called quasiprimitive if every nontrivial normal subgroup of $G$ acts transitively on $\Omega$. This is a generalisation of primitivity as every normal subgroup of a primitive group is transitive, but there exist quasiprimitive groups which are not primitive. The third author 23 generalised the O'Nan-Scott Theorem for primitive groups to quasiprimitive groups and showed that a finite quasiprimitive group is one of eight types. We now provide a description of these types along the lines of that in [24]. Throughout, T will be a finite nonabelian simple group.

The first three types of quasiprimitive groups are subgroups of the holomorph $\operatorname{Hol}(N)$ of a certain group $N$. This is defined as the semidirect product $\operatorname{Hol}(N)=$ $N \cdot \operatorname{Aut}(N)$ acting on $\Omega=N$ where, for all $n \in N$ and $\sigma \in \operatorname{Aut}(N)$,

$$
n \sigma: x \mapsto x^{\sigma} n^{\sigma} \text { for all } x \in \Omega \text {. }
$$


Thus the group $N$ is normal in $\operatorname{Hol}(N)$ and acts regularly by right multiplication.

The first quasiprimitive type is HA, and all groups of this type are in fact primitive. This class consists of all quasiprimitive groups with an elementary abelian minimal normal subgroup. Such groups are subgroups of $\operatorname{AGL}(d, p)$, for some prime $p$ and positive integer $d$, acting on the points of the affine space $\operatorname{AG}(d, p)$. The group $N \cong C_{p}^{d}$ of all translations is the unique minimal normal subgroup of $G$ and $G=N: G_{0}$ for some irreducible subgroup $G_{0} \leqslant \operatorname{GL}(d, p)$. Note that $\operatorname{AGL}(d, p)=\operatorname{Hol}(N)$.

The next type of quasiprimitive group is HS. Groups of this type are subgroups of the holomorph $\operatorname{Hol}(T)=T$. Aut $(T)$ containing $T \cdot \operatorname{Inn}(T)$ and acting on $\Omega=T$ as above. Such groups have two minimal normal subgroups, both of which are isomorphic to $T$ and act regularly on $\Omega$. One minimal normal subgroup is $T$ in its action on itself by right multiplication and the other is $T$ acting on itself by left multiplication. Thus the socle, $\operatorname{soc}(G)$, of $G$ is $T \times T=T$. $\operatorname{Inn}(T)$ and acts on $\Omega$ by

$$
\left(t_{1}, t_{2}\right): s \mapsto t_{2}^{-1} s t_{1} \text { for all } s \in T .
$$

All groups of this type are primitive.

The third type of quasiprimitive group $\mathrm{HC}$ also consists only of primitive groups. These groups are similar to those of type HS, but here $G \leqslant \operatorname{Hol}\left(T^{k}\right)$ for $k \geq 2$. The action on $\Omega=T^{k}$ is again the holomorph action and $G$ has two minimal normal subgroups, both regular and isomorphic to $T^{k}$. Now $\operatorname{Inn}\left(T^{k}\right) \leqslant G_{1} \leqslant \operatorname{Aut}\left(T^{k}\right)$ and we require that $G_{1}$ acts transitively on the $k$ simple direct factors of $T^{k}$. The groups of types $\mathrm{HS}$ and $\mathrm{HC}$ are the only quasiprimitive groups with two minimal normal subgroups. All the rest have only one.

We now define a twisted wreath product by following the description in [28] p. 269]. Let $T$ be a finite nonabelian simple group, $P$ a group with a proper subgroup $Q$ and let $\phi: Q \rightarrow T$ be a homomorphism. We define the complete base group $\mathcal{B}$ to be the set of maps $f: P \rightarrow T$ under pointwise multiplication and so $\mathcal{B} \cong T^{|P|}$. The group $P$ acts on $\mathcal{B}$ by

$$
f^{p}(x)=f(p x) \text { for all } x, p \in P, f \in \mathcal{B} .
$$

We define $\mathcal{X}$ to be the semidirect product $\mathcal{B}: P$ with respect to this action. Define the $\phi$-base group

$$
B_{\phi}=\left\{f: P \rightarrow T \mid f(p q)=f(p)^{\phi(q)} \text { for all } p \in P, q \in Q\right\} .
$$

This group is isomorphic to $T^{k}$, where $k=|P: Q|$. Also $B_{\phi}$ is normalised by $P$, and so $B_{\phi}$ and $P$ generate the subgroup $X_{\phi}=B_{\phi}: P$ of $\mathcal{X}$ which we call the twisted wreath product $T \operatorname{twr}_{\phi} P$ of $T$ by $P$ with respect to $\phi$. The action of $X_{\phi}$ on its base group is quasiprimitive if and only if $\phi^{-1}(\operatorname{Inn}(T))$ is a core free subgroup of $P$ (see [23]). Such groups are said to be of quasiprimitive type TW and are the only quasiprimitive groups with a unique minimal normal subgroup isomorphic to $T^{k}$ for $k \geq 2$ which acts regularly. The action is primitive if and only if $\phi$ does not extend to a larger subgroup of $P$ and $\operatorname{Inn}(T) \leqslant \phi(Q)$ (see [21]).

The next type of quasiprimitive group is AS, and these are all the almost simple groups with transitive socle $T$, that is $T \leqslant G \leqslant \operatorname{Aut}(T)$. We are not given any further information about the action in this case, and in particular it is possible for $T$ to act regularly on $\Omega$. The group $G$ is primitive if and only if a point stabiliser is a maximal subgroup of $G$ not containing $T$. 
Next we describe quasiprimitive groups of type SD. Let $N=T^{k}$ for $k \geq 2$. A full diagonal subgroup of $N$ is a subgroup isomorphic to $T$ whose projection onto every coordinate is also isomorphic to $T$. We let $N$ act on the set $\Omega$ of right cosets of the full diagonal subgroup $N_{\alpha}=\{(t, \ldots, t): t \in T\}$. Then the set $\left\{\left[t_{1}, \ldots, t_{k-1}, 1\right]: t_{i} \in T\right\}$ forms a set of coset representatives for $N_{\alpha}$ in $N$ and so we may identify $\Omega$ with $T^{k-1}$. The action of $N$ is then given by

$$
\left(s_{1}, \ldots, s_{k}\right):\left[t_{1}, \ldots, t_{k-1}, 1\right] \mapsto\left[s_{k}^{-1} t_{1} s_{1}, \ldots, s_{k}^{-1} t_{k-1} s_{k-1}, 1\right] .
$$

Let $W$ be the normaliser of $N$ in $\operatorname{Sym}(\Omega)$. Then for each $h \in \operatorname{Aut}(T)$, the permutation

$$
\left[t_{1}, \ldots, t_{k-1}, 1\right] \mapsto\left[t_{1}^{h}, \ldots, t_{k-1}^{h}, 1\right]
$$

induced by $h$ is in $W$. Note that the permutations induced by $\operatorname{Inn}(T)$ are precisely those induced by $N_{\alpha}$. Also, for each $\sigma \in S_{k}$, if we let $t_{k}=1$, the permutation

$$
\left[t_{1}, \ldots, t_{k-1}, 1\right] \mapsto\left[t_{1 \sigma^{-1}}, \ldots, t_{k \sigma^{-1}}\right]=\left[t_{k \sigma^{-1}}^{-1} t_{1 \sigma^{-1}}, \ldots, t_{k \sigma^{-1}}^{-1} t_{(k-1) \sigma^{-1}}, 1\right]
$$

is in $W$. In fact, $W=\left\langle N, \operatorname{Aut}(T), S_{k}\right\rangle \cong T^{k}$. $\left(\operatorname{Out}(T) \times S_{k}\right)$, where the extension of $T^{k}$ by $\operatorname{Out}(T) \times S_{k}$ is not necessarily a split extension. A quasiprimitive group of type SD is a group $G$ such that $N \triangleleft G \leqslant W$ and $G$ acts transitively by conjugation on the set of simple direct factors of $N$, that is, $N$ is the unique minimal normal subgroup of $G$. Such groups are primitive if and only if $G$ acts primitively on the $k$ simple direct factors of $N$. We note that, in the $k=2$ case, if $N \triangleleft G<W$ and $G$ does not act transitively by conjugation on the two simple direct factors of $N$, then $N$ is the product of two minimal normal subgroups of $G$ and $G$ is primitive of type HS.

Quasiprimitive groups of type CD are built from quasiprimitive groups of type SD. Let $H$ be a quasiprimitive group of type SD on the set $\Delta$ with a unique minimal normal subgroup $T^{l}$. For a positive integer $k$ divisible by $l$, if $G$ satisfies $N=T^{k} \leqslant G \leqslant H$ wr $S_{k / l}$, then $G$ acts on the set $\Omega=\Delta^{k / l}$ with the product action of the wreath product, namely, for $\left(\delta_{1}, \ldots, \delta_{k / l}\right) \in \Omega, h=\left(h_{1}, \ldots, h_{k / l}\right) \in H^{k / l}$ and $\sigma \in S_{k / l}$

$$
\left(\delta_{1}, \ldots, \delta_{k / l}\right)^{h}=\left(\delta_{1}^{h_{1}}, \ldots, \delta_{k / l}^{h_{k / l}}\right)
$$

and

$$
\left(\delta_{1}, \ldots, \delta_{k / l}\right)^{\sigma}=\left(\delta_{1 \sigma^{-1}}, \ldots, \delta_{(k / l) \sigma^{-1}}\right) .
$$

This action is quasiprimitive if and only if $G$ acts transitively by conjugation on the set of simple direct factors of $N$. Then $N$ is the unique minimal normal subgroup of $G$. The action is primitive if $H$ is primitive. We note here that groups of type $\mathrm{HC}$ can be built up in the same way from groups of type HS.

The last type of quasiprimitive group is PA. A group $G$ of this type preserves some partition $\mathcal{P}$ (possibly trivial in the sense of having parts of size 1 ) of $\Omega$ upon which $G$ acts faithfully preserving a product structure $\Delta^{k}$. Furthermore, $N=T^{k} \leqslant G \leqslant H$ wr $S_{k}$, where $H$ acts quasiprimitively on $\Delta$ of type AS with nonregular socle $T$ and $G$ acts transitively by conjugation on the set of simple direct factors of $N$. Let $\delta \in \Delta$ and $B=(\delta, \ldots, \delta) \in \mathcal{P}$. Then $N_{B}=T_{\delta}^{k}$ and for $\omega \in B$, the point stabiliser $N_{\omega}$ is a subdirect subgroup of $N_{B}$, that is, $N_{\omega}$ projects onto $T_{\delta}$ in every coordinate. The action of $G$ is primitive if and only if $\mathcal{P}$ is trivial and the action of $H$ on $\Delta$ is primitive. 


\section{General graph COnstructions}

In this section we provide three methods for constructing $G$-edge transitive graphs. The constructions in Sections 3.1 and 3.3 enable us to build a locally $(G, s)$-arc transitive graph from a given $(G, s)$-arc transitive graph. Section 3.2 shows how to construct arbitrary locally $(G, s)$-arc transitive, $G$-vertex intransitive graphs as coset graphs. The construction in Section 3.3 characterises locally $(G, s)$-arc transitive graphs with a vertex of valency two.

We use the following conventions for graphs. A digraph $\Gamma$ consists of a vertex set $V \Gamma$ and a subset $A \Gamma$ of $(V \Gamma \times V \Gamma) \backslash\{(v, v): v \in V \Gamma\}$ called arcs. If $A \Gamma$ is self-paired in the sense that $(x, y) \in A \Gamma$ if and only if $(y, x) \in A \Gamma$, then we say that $\Gamma$ is self-paired. In this case we usually replace every pair of arcs between two vertices with a single undirected edge to form an undirected graph. The edge set is denoted $E \Gamma$. If $G \leqslant \operatorname{Aut}(\Gamma)$ is edge transitive on $\Gamma$ with two vertex orbits $\Delta_{1}$ and $\Delta_{2}$, then all vertices in the same orbit $\Delta_{i}$ have the same valency $k_{i}$ say, and we say that $\Gamma$ is biregular of valency $\left\{k_{1}, k_{2}\right\}$. If $k_{1}=k_{2}=k$, then $\Gamma$ is regular of valency $k$.

Let $G$ be a transitive permutation group on the set $V$ and let $\mathcal{O}$ be a nontrivial orbital for $G$, that is, an orbit of $G$ on $(V \times V) \backslash\{(v, v): v \in V\}$. We define the orbital digraph $\Gamma$ for $G$ with respect to $\mathcal{O}$ to be the directed graph with vertex set $V$ and arc set $\mathcal{O}$. This graph is $G$-vertex transitive and $G$-arc transitive. Conversely, every $G$-vertex transitive and $G$-arc transitive graph can be constructed in this way. If $\mathcal{O}$ is self-paired, then the corresponding undirected graph is called an orbital graph.

We note the following elementary lemmas. The proof of the first can be found, for example, in [2].

Lemma 3.1. Let $\Gamma$ be a connected locally $(G, s)$-arc transitive graph such that $s \geq 1$ and all vertices have valency at least two. Then $G$ acts transitively on the set of edges of $\Gamma$. Furthermore, if $G$ acts intranstively on $V \Gamma$, then $\Gamma$ is a bipartite graph and the two parts of the bipartition are $G$-orbits.

Lemma 3.2. Let $\Gamma$ be a graph such that all vertices have valency at least two. Then $\Gamma$ is locally $(G, 2)$-arc transitive if and only if for every vertex $v, G_{v}$ acts 2-transitively on $\Gamma(v)$.

Proof. Let $\Gamma$ be locally $(G, 2)$-arc transitive with all vertices having valency at least two and let $v \in V \Gamma$. By Lemma 3.1 $G$ acts transitively on the set of edges of $\Gamma$ and so $G_{v}$ acts transitively on $\Gamma(v)$. Let $u \in \Gamma(v)$. Then for every $w \in \Gamma(v) \backslash\{u\}$ there is a $2-\operatorname{arc}(u, v, w)$. As $\Gamma$ is locally $(G, 2)-\operatorname{arc}$ transitive, it follows that $G_{u v}$ acts transitively on $\Gamma(v) \backslash\{u\}$ and so $G_{v}$ acts 2 -transitively on $\Gamma(v)$.

Conversely, suppose that for every vertex $v, G_{v}$ acts 2 -transitively on $\Gamma(v)$. Let $\left(v, u_{1}, w_{1}\right)$ and $\left(v, u_{2}, w_{2}\right)$ be two $2-\operatorname{arcs}$ in $\Gamma$. Then as $G_{v}$ acts transitively on $\Gamma(v)$, there exists $g \in G_{v}$ such that $\left(v, u_{1}, w_{1}\right)^{g}=\left(v, u_{2}, w_{1}^{\prime}\right)$ for some $w_{1}^{\prime} \in \Gamma\left(u_{2}\right) \backslash\{v\}$. Also, as $G_{u_{2}}$ acts 2 -transitively on $\Gamma\left(u_{2}\right)$, there exists $h \in G_{u_{2} v}$ such that $\left(w_{1}^{\prime}\right)^{h}=$ $w_{2}$. Hence $\left(v, u_{1}, w_{1}\right)^{g h}=\left(v, u_{2}, w_{2}\right)$ and so $\Gamma$ is locally $(G, 2)$-arc transitive.

3.1. Double covers. Let $\Gamma$ be a directed or undirected vertex transitive graph with vertex set $V \Gamma$ and arc set $A \Gamma$. The standard double cover of $\Gamma$ is the undirected graph $\bar{\Gamma}$ with a vertex set $V \Gamma \times\{1,2\}$, and two vertices $(x, 1)$ and $(y, 2)$ are adjacent if and only $(x, y) \in A \Gamma$. The new graph is bipartite with bipartite halves $V \Gamma \times\{i\}$ for each $i=1,2$. 
Now if $G \leqslant \operatorname{Aut}(\Gamma)$, then $G$ also acts as a group of automorphisms of $\bar{\Gamma}$ with the action $g:(x, i) \mapsto\left(x^{g}, i\right)$. If $G$ is vertex transitive on $\Gamma$, then $G$ has two orbits on the set of vertices of $\bar{\Gamma}$ and the action of $G$ on each orbit is permutationally isomorphic to the action of $G$ on $V \Gamma$. Furthermore, $G_{v}=G_{(v, i)}$ for each $i=1,2$. Then if $\Gamma$ is undirected, the action of $G_{v}$ on $\Gamma(v)$ is the same as the action of $G_{(v, i)}$ on $\bar{\Gamma}((v, i))$. Thus in this case, if $\Gamma$ is $G$-locally primitive, $\bar{\Gamma}$ is also $G$-locally primitive.

Suppose again that $\Gamma$ is undirected. Then $(x, 1) \sim(y, 2)$ if and only if $(y, 1) \sim$ $(x, 2)$. If $\Gamma$ is also connected, then for each $x, y \in V \Gamma$ there exists a path $P$ in $\Gamma$ between $x$ and $y$. This path lifts to a path in $\bar{\Gamma}$ between $(x, 1)$ and $(y, 1)$ if $P$ has even length, and to one between $(x, 1)$ and $(y, 2)$ if $P$ has odd length. There is a path between $(y, 1)$ and $(y, 2)$ if and only if $y$ is in an odd cycle in $\Gamma$. Thus for an undirected connected graph $\Gamma, \bar{\Gamma}$ is connected if and only if $\Gamma$ contains an odd cycle, that is, if and only if $\Gamma$ is not bipartite. If $\left((v, i),\left(v_{1}, j\right), \ldots,\left(v_{s}, i\right)\right)$ is an $s$-arc in $\bar{\Gamma}$, then $\left(v, v_{1}, \ldots, v_{s}\right)$ is an $s$-arc in $\Gamma$. Thus if $\Gamma$ is $(G, s)$-arc transitive, then $\bar{\Gamma}$ is locally $(G, s)$-arc transitive. Since there are nonbipartite, $(G, 2)$-arc transitive graphs with $G$ quasiprimitive of each of the types HA, TW, AS and PA (see [24]), we can construct locally $(G, 2)$-arc transitive graphs with $G$ of types HA, TW, AS and PA on both of its orbits by taking their standard double covers. Note that these graphs are Aut $(\Gamma)$-vertex transitive, as the map $\tau:(x, i) \mapsto(x, 3-i)$ is an automorphism interchanging the two halves of the bipartition. We summarise this discussion in the following lemma.

Lemma 3.3. Let $\Gamma$ be an undirected graph. Then

(1) $\bar{\Gamma}$ is connected if and only if $\Gamma$ is connected and not bipartite.

(2) If $\Gamma$ is $G$-locally primitive, then $\bar{\Gamma}$ is G-locally primitive.

(3) If $\Gamma$ is $(G, s)$-arc transitive, then $\bar{\Gamma}$ is locally $(G, s)$-arc transitive. In particular, there exist quasiprimitive locally $(G, 2)$-arc transitive graphs of types HA, TW, AS and PA.

Note that when $\Gamma$ is directed, paths and $s-\operatorname{arcs}$ in $\Gamma$ no longer lift to paths and $s-\operatorname{arcs}$ in $\bar{\Gamma}$.

Now let $\Gamma$ be a $G$-edge transitive, bipartite graph such that each vertex has valency at least two, and suppose that $G$ has two orbits $\Delta_{1}$ and $\Delta_{2}$ on vertices and all vertex stabilisers are conjugate. Then the two actions of $G$ are permutationally isomorphic and so there exists a bijection $\phi: \Delta_{1} \rightarrow \Delta_{2}$ such that $\phi\left(v^{g}\right)=\phi(v)^{g}$ for all $v \in \Delta_{1}$ and $g \in G$. Note that $v$ and $\phi(v)$ have the same vertex stabilisers. By identifying the vertices $v$ and $\phi(v)$, we can label the vertex set of $\Gamma$ with $\Delta_{1} \times\{1,2\}$. Define the directed graph $\Sigma$ with vertex set $\Delta_{1}$ and $\operatorname{arc}$ set such that $(v, w)$ is an arc if and only if $(v, 1) \sim(w, 2)$ in $\Gamma$. Note that $(v, 1)$ is not adjacent to $(v, 2)$ in $\Gamma$ as $G_{(v, 1)}$ acts transitively on edges emerging from $(v, 1)$. Then $\Sigma$ is a $G$-vertex transitive and $G$-arc transitive directed graph and so is an orbital digraph for $G$ on $\Delta_{1}$. Furthermore, $\Gamma$ is the standard double cover of the directed graph $\Sigma$. The bijection $\varphi: V \Gamma \rightarrow V \Gamma$ which interchanges $(v, 1)$ and $(v, 2)$ for all $v \in \Delta_{1}$ is an automorphism for $\Gamma$ if and only if for every $\operatorname{arc}(v, w)$ in $\Sigma,(w, v)$ is also an arc. Thus we have the following.

Lemma 3.4. Let $\Gamma$ be a $G$-edge transitive graph of valency at least two with two vertex orbits $\Delta_{1}$ and $\Delta_{2}$ such that all vertex stabilisers are conjugate. Then $\Gamma$ is the standard double cover of an orbital digraph $\Sigma$ for $G$ on $\Delta_{1}$, and the bijection 
which interchanges $(v, 1)$ with $(v, 2)$ is an automorphism for $\Gamma$ if and only if $\Sigma$ is self paired.

Another condition for such a graph $\Gamma$ to be $\operatorname{Aut}(\Gamma)$-vertex transitive is given in [10. Lemma 2.5]. There are examples in [10] of locally $(G, s)$-arc transitive graphs for which all vertex stabilisers are conjugate in $G$, yet $\operatorname{Aut}(\Gamma)$ is not vertex transitive.

3.2. Coset graphs. Let $\Gamma$ be a $G$-edge transitive graph with vertex set $V \Gamma$, where $G \leqslant \operatorname{Aut}(\Gamma)$. Assume that $G$ is not transitive on $V \Gamma$. Then by Lemma $3.1 \Gamma$ is a bipartite graph with parts $\Delta_{1}$ and $\Delta_{2}$ such that $G$ is transitive on both $\Delta_{1}$ and $\Delta_{2}$. Since $G$ is transitive on $\Delta_{1}$, for $w \in \Delta_{1}$ we may write $\Delta_{1}$ as the set $\left[G: G_{v}\right.$ ] of right cosets of $G_{v}$ in $G$ so that $G$ acts transitively on $\Delta_{1}$ by right multiplication, that is,

$$
\begin{aligned}
& \Delta_{1}=\left\{G_{v} x: x \in G\right\}, \text { and } \\
& z: G_{v} x \rightarrow G_{v} x z, \text { for each } z \in G .
\end{aligned}
$$

Similarly, for $w \in \Delta_{2}$, we may write $\Delta_{2}$ as the set $\left[G: G_{w}\right]$ of right cosets of $G_{w}$ in $G$ so that $G$ acts transitively on $\Delta_{2}$ by right multiplication, that is,

$$
\begin{aligned}
& \Delta_{2}=\left\{G_{w} x: x \in G\right\} \text { and } \\
& z: G_{w} x \rightarrow G_{w} x z, \text { for each } z \in G .
\end{aligned}
$$

Thus the vertices of $\Gamma$ may be identified with right cosets of $G_{v}$ and $G_{w}$ in $G$. Now assume that $v$ is adjacent to $w$. Then $G_{v} \cap G_{w}$ is the stabiliser of the edge $\{v, w\}$ in $G$. It follows since $G \leqslant \operatorname{Aut}(\Gamma)$ that $G_{v} \cap G_{w}$ is core free in $G$. Since $G_{v}$ is transitive on $\Gamma(v)$ (by right multiplication), the neighbours of $v$ are exactly the images of $w$ under $G_{v}$, that is $\left\{G_{w} z: z \in G_{v}\right\}$. Similarly, the neighbours of $w$ may be identified with $\left\{G_{v} z: z \in G_{w}\right\}$. The adjacency relation of $\Gamma$ is given by

$$
G_{v} x \sim G_{w} y \Longleftrightarrow x y^{-1} \in G_{v} G_{w} \text {, or equivalently } y x^{-1} \in G_{w} G_{v} .
$$

Hence the vertex set and the adjacency relation of the graph $\Gamma$ can be explicitly described in terms of cosets of the point stabilisers. Thus we have the following result.

Lemma 3.5. Let $\Gamma$ be a bipartite $G$-edge transitive graph with parts $\Delta_{1}$ and $\Delta_{2}$, where $G \leqslant \operatorname{Aut}(\Gamma)$ and $G$ is intransitive on $V \Gamma$. Let $v \in \Delta_{1}$ and $w \in \Delta_{2}$ be adjacent. Then we may identify $\Delta_{1}=\left[G: G_{v}\right]$ and $\Delta_{2}=\left[G: G_{w}\right]$ such that

(1) $G_{v} x$ is adjacent to $G_{w} y$ if and only if $x y^{-1} \in G_{v} G_{w}$, or equivalently, $y x^{-1} \in$ $G_{w} G_{v}$

(2) $\Gamma(v)=\left\{G_{w} z: z \in G_{v}\right\}=G_{w} G_{v}$ and $\Gamma(w)=\left\{G_{v} z: z \in G_{w}\right\}=G_{v} G_{w}$;

(3) the valencies are $|\Gamma(v)|=\left|G_{v}: G_{v} \cap G_{w}\right|$ and $|\Gamma(w)|=\left|G_{w}: G_{v} \cap G_{w}\right|$.

Conversely, we may construct edge transitive graphs from any given abstract group.

Definition 3.6. Let $G$ be a group, and let $L, R<G$ be such that $L \cap R$ is core free in $G$. Let $\Delta_{1}=\{L x: x \in G\}$ and $\Delta_{2}=\{R y: y \in G\}$. Define the bipartite graph

$$
\Gamma=\operatorname{Cos}(G, L, R)
$$

to be the graph with vertex set $V=\Delta_{1} \dot{\cup} \Delta_{2}$ such that

$$
L x \sim R y \Longleftrightarrow x y^{-1} \in L R \text {, or equivalently } y x^{-1} \in R L .
$$

We refer to $(L, R, L \cap R)$ as the associated amalgam. 
We note that $x y^{-1} \in L R$ if and only if $L x \cap R y \neq \varnothing$, so the above definition of coset graphs coincides with Tits's construction of flag transitive incidence structures; see [7]. It is also related to $\mathrm{Du}$ and Xu's [9] construction of bi-coset graphs. The next simple lemma gives some properties of the graphs $\operatorname{Cos}(G, L, R)$.

Lemma 3.7. For a group $G$ and subgroups $L, R<G$ such that $L \cap R$ is core free in $G$, the graph $\Gamma=\operatorname{Cos}(G, L, R)$ satisfies the following properties:

(1) $\Gamma$ is connected if and only if $\langle L, R\rangle=G$;

(2) $G \leqslant \operatorname{Aut}(\Gamma)$, and $\Gamma$ is $G$-edge transitive and $G$-vertex intransitive;

(3) $G$ acts faithfully on both $\Delta_{1}$ and $\Delta_{2}$ if and only if both $L$ and $R$ are core free;

(4) $\Gamma$ is $G$-locally primitive if and only if $L \cap R$ is a maximal subgroup of both $L$ and $R$;

(5) $\Gamma$ is locally $(G, 2)$-arc transitive if and only if $L$ acts 2 -transitively on $[L$ : $L \cap R]$ and $R$ acts 2-transitively on $[R: L \cap R]$.

Conversely, if $\Gamma$ is a $G$-edge transitive but not $G$-vertex transitive graph, and $v$ and $w$ are adjacent vertices, then $\Gamma \cong \operatorname{Cos}\left(G, G_{v}, G_{w}\right)$.

Proof. (1) Assume that $G=\langle L, R\rangle$. Then for any $w \in G, w=x_{1} y_{1} \ldots x_{k} y_{k}$ for some $x_{i} \in L$ and some $y_{i} \in R$ such that $x_{i} \neq 1$ if $i \neq 1$, and $y_{i} \neq 1$ if $i \neq k$. There is a path in $\Gamma$ from $L$ to $L w$ :

$$
L, R y_{k}, L x_{k} y_{k}, \ldots, R y_{1} \ldots x_{k} y_{k}, L x_{1} y_{1} \ldots x_{k} y_{k}=L w .
$$

There is also a path in $\Gamma$ from $L$ to $R w$ :

$$
L, R, L y_{k}, R x_{k} y_{k}, \ldots, L y_{1} \ldots x_{k} y_{k}, R x_{1} y_{1} \ldots x_{k} y_{k}=R w .
$$

Thus $\Gamma$ is connected. Conversely, if $\Gamma$ is connected, it is easily shown that $G=$ $\langle L, R\rangle$.

(2) Let $\Delta_{1}=\{L x: x \in G\}$ and $\Delta_{2}=\{R y: y \in G\}$. For $z \in G$, let $\hat{z}$ be the permutation on $V=\Delta_{1} \cup \Delta_{2}$ induced by $z$ defined as

$$
\hat{z}: \quad L x \rightarrow L x z, \quad R y \rightarrow R y z .
$$

Then

$$
\begin{aligned}
L x \sim R y & \Longleftrightarrow x y^{-1} \in L R \\
& \Longleftrightarrow x z(y z)^{-1}=x z z^{-1} y^{-1} \in L R \\
& \Longleftrightarrow L x z \sim R y z \\
& \Longleftrightarrow(L x)^{\hat{z}} \sim(R y)^{\hat{z}} .
\end{aligned}
$$

Thus $\hat{z}$ is an automorphism of $\Gamma$, and so there is a homomorphism $\phi: G \rightarrow \operatorname{Aut}(\Gamma)$ given by $\phi(z)=\hat{z}$. The kernel of this homomorphism is trivial since $L \cap R$ is core free and hence $G \leqslant \operatorname{Aut}(\Gamma)$. Clearly, no element of $G$ maps $L \in \Delta_{1}$ to $R \in \Delta_{2}$, and hence $G$ is intransitive on $V$.

Write $v$ and $w$ to be the vertices corresponding to $L$ and $R$, respectively. By definition, the set of neighbours of $v$ equals $\{R x: x \in L\}$. For any $z \in L, L^{\hat{z}}=$ $L z=L$ and $R^{\hat{z}}=R z \in\{R y: y \in L\}=\Gamma(v)$. When $z$ runs through $L, R z$ runs through $\{R y: y \in L\}$. Therefore, $L$ is transitive on $\Gamma(v)$. Similarly, $R$ is transitive on $\Gamma(w)=\{L x: x \in G\}$ and so $\Gamma$ is $G$-edge transitive.

(3) This follows as $G_{v}=L$ and $G_{w}=R$.

(4) This follows as $G_{v w}=G_{w} \cap G_{v}=L \cap R$.

(5) This follows from Lemma 3.2 . 
Finally, let $\Gamma$ be any $G$-edge transitive graph that is not $G$-vertex transitive and let $v$ and $w$ be adjacent vertices. The fact that $\Gamma \cong \operatorname{Cos}\left(G, G_{v}, G_{w}\right)$ follows from Lemma [3.5 and Definition 3.6

Given a group $G$ with subgroup $L$ and element $g$ we can also form the coset digraph $\Sigma=\operatorname{Cos}(G, L, L g L)$ which has vertex set $[G: L]$ and $(L x, L y) \in A \Sigma$ if and only if $y x^{-1} \in L g L$. This directed graph is connected if and only if $\langle L, g\rangle=G$ and is self paired if and only if $g^{-1} \in L g L$. Furthermore, $G$ acts as an arc transitive group of automorphisms by right multiplication on the cosets. This construction is due to Sabidussi [25] and is related to our coset construction in the following way.

Lemma 3.8. Let $G$ be a group with subgroup $L$ and element $g$ such that $L \cap L^{g}$ is core free. Then $\Gamma=\operatorname{Cos}\left(G, L, L^{g}\right)$ is the standard double cover of

$$
\Sigma=\operatorname{Cos}(G, L, L g L) \text {. }
$$

Proof. Let $\bar{\Sigma}$ be the standard double cover of $\Sigma$ and define the map

$$
\begin{aligned}
\phi: \quad V \bar{\Sigma} & \rightarrow V \Gamma, \\
(L x, 1) & \mapsto L x, \\
(L x, 2) & \mapsto L^{g} g^{-1} x .
\end{aligned}
$$

Let $g_{1}, g_{2} \in G$. Then

$$
\begin{aligned}
L^{g} g^{-1} g_{1}=L^{g} g^{-1} g_{2} & \Longleftrightarrow g^{-1} g_{1} g_{2}^{-1} g \in L^{g} \\
& \Longleftrightarrow g_{1} g_{2}^{-1} \in L \\
& \Longleftrightarrow L g_{1}=L g_{2} .
\end{aligned}
$$

Furthermore, if $\mathcal{T}$ is a transversal for $L$ in $G$, then $g^{-1} \mathcal{T}$ is a transversal for $L^{g}$ in $G$. Thus $\phi$ is a well-defined bijection. Suppose that $(L x, 1) \sim(L y, 2)$ in $\bar{\Sigma}$. Then $(L x, L y)$ is an arc in $\Sigma$ and so $y x^{-1} \in L g L$. Hence $x y^{-1} \in L g^{-1} L$ and so $x y^{-1} g \in$ $L g^{-1} L g=L L^{g}$. Thus $\phi((L x, 1))=L x$ is adjacent to $\phi((L y, 2))=L^{g} g^{-1} y$ in $\Gamma$. Conversely, suppose that $\left(L x, L^{g} y\right) \in E \Gamma$. Then $x y^{-1} \in L L^{g}$ and so $y x^{-1} \in L^{g} L$. Now $\phi((L x, 1))=L x$ and $\phi((L g y, 2))=L^{g} y$. Furthermore, $g y x^{-1} \in g L^{g} L=L g L$ and so $(L x, 1) \sim(L g y, 2)$ in $\bar{\Sigma}$. Thus $\phi$ is a graph isomorphism.

A version of Lemma 3.8 was proved in [10, Lemma 2.6] in the case where $g^{2} \in L$.

3.3. Graphs with a vertex of valency one or two. If $\Gamma$ is a locally $(G, s)$-arc transitive graph with all vertices of valency at least two, then $\Gamma$ is $(G, s-1)$-arc transitive. Furthermore, if $G$ acts intransitively on vertices, then $\Gamma$ is bipartite with the two parts of the bipartition being $G$-orbits. However, if $\Gamma$ has a vertex of valency one, then we cannot make these two deductions. We have the following lemma.

Lemma 3.9. Let $\Gamma$ be a connected locally $(G, s)$-arc transitive graph with $s \geq 1$ which contains a vertex of valency one. Then $\Gamma$ is a tree.

Proof. Let $v$ be a vertex of valency one in $\Gamma$ and suppose that $\Gamma$ contains a cycle. Then as $\Gamma$ is connected, there exists a shortest path $\left\{w_{0}=v, w_{1}, \ldots, w_{t}\right\}$ such that $w_{t}$ is contained in a cycle $C$. Since $v$ has valency one, we have $t \geq 1$ and none of the vertices $w_{0}, w_{1}, \ldots, w_{t-1}$ lies on a cycle. There is an $s$-arc that starts with the $\operatorname{arc}\left(w_{t-1}, w_{t}\right)$ and then proceeds to loop around $C$ finishing at a vertex $u \in C$. Let $\beta$ be the reverse of this $s$-arc running from $u$ to $w_{t-1}$. Let $\alpha$ be the $s-\operatorname{arc}$ that agrees with $\beta$ in its first $s$ vertices but ends in a vertex of $C$ adjacent to $w_{t}$. As 
$w_{t-1} \in \beta$ and is not contained in a cycle while all vertices in $\alpha$ belong to $C$, there is no element of $G_{u}$ mapping $\alpha$ to $\beta$. This contradicts $\Gamma$ being locally $(G, s)-\operatorname{arc}$ transitive and so $\Gamma$ is a tree.

Let $\Gamma$ be a $(G, s)$-arc transitive graph with valency $k \geq 2$. We follow [2] and form a new graph $\Gamma^{*}$ by placing a vertex at the midpoint of each edge of $\Gamma$. Then $G \leqslant \operatorname{Aut}\left(\Gamma^{*}\right)$ and has two orbits on vertices: the set $\Delta_{1}$ of vertices of $\Gamma$ and the set $\Delta_{2}$ of midpoints of edges of $\Gamma$. All vertices in $\Delta_{1}$ have valency $k$ while those in $\Delta_{2}$ have valency two. If $\Gamma$ is a cycle, then the new graph $\Gamma^{*}$ is also a cycle. We will show in Theorem 3.10 that $\Gamma^{*}$ is locally $(G, 2 s-1)$-arc transitive.

In fact every locally $(G, 2 s-1)$-arc transitive graph with a vertex of valency two can be constructed in this way. First we need a definition. Given a connected graph $\Gamma$, the distance two graph of $\Gamma$ is the graph with vertex set $V \Gamma$ such that two vertices are adjacent if and only if they are at distance two in $\Gamma$. Note that if $\Gamma$ is bipartite, then the distance two graph of $\Gamma$ has two connected components. Also $\Gamma$ is the connected component of the distance two graph of $\Gamma^{*}$ with vertex set $\Delta_{1}$.

Theorem 3.10. Let $s \geq 1$.

(a) Let $\Gamma$ be a connected locally $(G, 2 s-1)$-arc transitive graph of valency $\{2, k\}$ with $k \geq 3$ and let $\widetilde{\Gamma}$ be a connected component of the distance two graph of $\Gamma$ containing a vertex of valency $k$. Then $V \widetilde{\Gamma}$ is the set of all vertices of $\Gamma$ of valency $k$ and $\widetilde{\Gamma}$ is $(G, s)$-arc transitive of valency $k$.

(b) Let $\Sigma$ be a connected $(G, s)$-arc transitive graph of valency $k$. Then $\Sigma^{*}$ is a connected locally $(G, 2 s-1)$-arc transitive graph. Moreover, if $\Gamma=\Sigma^{*}$, then the graph $\widetilde{\Gamma}$ in part (a) is equal to $\Sigma$.

Proof. (a) Let $\Gamma$ be a connected locally $(G, 2 s-1)$-arc transitive graph such that $G$ has two orbits $\Delta_{1}$ and $\Delta_{2}$ on vertices. Suppose that all vertices in $\Delta_{2}$ have valency two while the vertices in $\Delta_{1}$ have valency $k \geq 3$. Let $\widetilde{\Gamma}$ be a connected component of the distance two graph of $\Gamma$ containing a vertex of valency $k$. Then as $\Gamma$ is connected, $V \widetilde{\Gamma}=\Delta_{1}$. Let $v \in \Delta_{1}$ and $w \in \Gamma(v)$. Then there exists a unique element $v(w) \in \Gamma(w) \backslash\{v\}$ and so the map $w \mapsto v(w)$ is a $1-1$ correspondence between $\Gamma(v)$ and $\Gamma_{2}(v)$. Thus $\widetilde{\Gamma}$ has valency $k$. Now $G \leqslant \operatorname{Aut}(\widetilde{\Gamma})$ and acts transitively on vertices. Let $\left(v_{0}, v_{1}, \ldots, v_{s}\right)$ be an $s$-arc in $\widetilde{\Gamma}$. Then for each $i=0, \ldots, s-1$ there exists a unique $w_{i}$ such that $\Gamma\left(w_{i}\right)=\left\{v_{i}, v_{i+1}\right\}$ and so $\left(v_{0}, w_{0}, v_{1}, \ldots, v_{s-1}, w_{s-1}\right)$ is a $(2 s-1)-\operatorname{arc}$ in $\Gamma$. Since $\Gamma$ is locally $(G, 2 s-1)$-arc transitive, it follows that $G_{v_{0}}$ is transitive on the set of all $s$-arcs in $\widetilde{\Gamma}$ beginning with $v_{0}$. As $\widetilde{\Gamma}$ is $G$-vertex transitive, this implies that $\widetilde{\Gamma}$ is $(G, s)$-arc transitive.

(b) Let $\Sigma$ be a connected $(G, s)$-arc transitive graph of valency $k$ and form the new graph $\Sigma^{*}$ by placing a new vertex at the midpoint of every edge. We have seen that $G \leqslant \operatorname{Aut}\left(\Sigma^{*}\right)$ and has two orbits $\Delta_{1}$ and $\Delta_{2}$ on vertices, with $\Delta_{1}$ being the set of all vertices of $\Sigma$ and $\Delta_{2}$ the set of midpoints. Let $v_{0} \in \Delta_{1}$ and consider the $(2 s-1)$-arc $\left(v_{0}, p_{0}, v_{1}, p_{1}, \ldots, v_{s-1}, p_{s-1}\right)$ in $\Sigma^{*}$, where each $p_{i}$ is the midpoint of the edge $\left\{v_{i}, v_{i+1}\right\}$. Then $\left(v_{0}, v_{1}, \ldots, v_{s}\right)$ is an $s$-arc in $\Sigma$. As $G_{v_{0}}$ acts transitively on the set of $s$-arcs in $\Sigma$ starting at $v_{0}$, it also acts transitively on the set of $(2 s-1)$-arcs in $\Sigma^{*}$ emanating from $v_{0}$. Thus to show that $\Sigma^{*}$ is locally $(G, 2 s-1)$-arc transitive it remains to show that for all $p_{0} \in \Delta_{2}, G_{p_{0}}$ acts transitively on the set of $(2 s-1)-\operatorname{arcs}$ starting at $p_{0}$. Given two such $(2 s-1)-\operatorname{arcs}$ $\alpha=\left(p_{0}, v_{1}, p_{1}, \ldots, p_{s-1}, v_{s}\right)$ and $\alpha^{\prime}=\left(p_{0}, v_{1}^{\prime}, p_{1}^{\prime}, \ldots, p_{s-1}^{\prime}, v_{s}^{\prime}\right)$ in $\Sigma^{*}$, there exist 
$s-\operatorname{arcs} \beta=\left(v_{0}, v_{1}, \ldots, v_{s}\right)$ and $\beta^{\prime}=\left(v_{0}^{\prime}, \ldots, v_{s}^{\prime}\right)$ in $\Sigma$ such that $p_{i}$ is the midpoint of $\left\{v_{i}, v_{i+1}\right\}$ and $p_{i}^{\prime}$ is the midpoint of $\left\{v_{i}^{\prime}, v_{i+1}^{\prime}\right\}$ for each $i \geq 0$. As $\Sigma$ is $(G, s)$-arc transitive, there exists $g \in G$ such that $\beta^{g}=\beta^{\prime}$ and hence $\alpha^{g}=\alpha^{\prime}$. However, $p_{0}$ is the midpoint of both $\left\{v_{0}, v_{1}\right\}$ and $\left\{v_{0}^{\prime}, v_{1}^{\prime}\right\}$, and so $\left\{v_{0}, v_{1}\right\}=\left\{v_{0}^{\prime}, v_{1}^{\prime}\right\}$. Hence $g \in G_{\left\{v_{0}, v_{1}\right\}}=G_{p_{0}}$. Thus $G_{p_{0}}$ acts transitively on the set of $(2 s-1)$-arcs starting at $p_{0}$, so $\Sigma^{*}$ is locally $(G, 2 s-1)$-arc transitive. The last assertion of (b) follows from the discussion immediately preceding the statement of the theorem.

Corollary 3.11. The connected locally $(G, 2 s-1)$-arc transitive graphs of valency $\{2, k\}$, where $k \geq 3$, are in 1-1 correspondence with the connected $(G, s)$-arc transitive graphs of valency $k$.

We now aim to give an upper bound for $s$ when $\Gamma$ has a vertex of valency two and a vertex of valency at least three.

Theorem 3.12. Let $\Gamma$ be a connected locally $(G, 2 s)$-arc transitive graph of valency $\{2, k\}$, where $k \geq 3$ and $s \geq 1$. Then $\Gamma$ is locally $(G, 2 s+1)$-arc transitive.

Proof. Let $\Delta_{1}$ and $\Delta_{2}$ denote the sets of vertices of valency $k$ and 2 , respectively. As all vertices in $\Gamma$ have valency at least two, $\Gamma$ is locally $(G, 2 s-1)-\operatorname{arc}$ transitive. Let $\widetilde{\Gamma}$ be the connected component of the distance two graph of $\Gamma$ with vertex set $\Delta_{1}$. Then by Theorem 3.10 (a), $\widetilde{\Gamma}$ is $(G, s)$-arc transitive. Let $\left(u_{0}, v_{1}, u_{1}, v_{2}, \ldots, v_{s}, u_{s}\right)$ be a $2 s-$ arc in $\Gamma$ where each $v_{i} \in \Delta_{1}$ and $u_{i} \in \Delta_{2}$. Now as $\Gamma$ is locally $(G, 2 s)-\operatorname{arc}$ transitive, $G_{u_{0} v_{1} u_{1} \ldots v_{s}}$ acts transitively on $\Gamma\left(v_{s}\right) \backslash\left\{u_{s-1}\right\}$. Then as each vertex of $\Gamma\left(v_{s}\right) \backslash\left\{u_{s-1}\right\}$ is adjacent to a unique vertex of $\Gamma_{2}\left(v_{s}\right) \backslash\left\{v_{s-1}\right\}$, it follows that $G_{u_{0} v_{1} u_{1} \ldots v_{s}}$ acts transitively on $\Gamma_{2}\left(v_{s}\right) \backslash\left\{v_{s-1}\right\}$. Now as $u_{0}$ has valency two, there exists a unique $v_{0} \in \Gamma\left(u_{0}\right) \backslash\left\{v_{1}\right\}$. Thus $G_{u_{0} v_{1} u_{1} \ldots v_{s}} \leqslant G_{v_{0}}$. Furthermore, $\left(v_{0}, v_{1}, \ldots, v_{s}\right)$ is an $s^{-}$ $\operatorname{arc}$ in $\widetilde{\Gamma}$ and $G_{v_{0} v_{1} \ldots v_{s}}$ acts transitively on $\widetilde{\Gamma}\left(v_{s}\right) \backslash\left\{v_{s-1}\right\}=\Gamma_{2}\left(v_{s}\right) \backslash\left\{v_{s-1}\right\}$. Hence $G_{v_{0}}$ acts transitively on the set of $(s+1)-\operatorname{arcs}$ in $\widetilde{\Gamma}$ starting at $v_{0}$ and so $\widetilde{\Gamma}$ is $(G, s+1)-\operatorname{arc}$ transitive. As $\Gamma=(\widetilde{\Gamma})^{*}$ it follows from Theorem 3.10 (b) that $\Gamma$ is locally $(2 s+1)-$ arc transitive.

Corollary 3.13. Let $\Gamma$ be a locally $(G, s)$-arc transitive graph of valency $\{2, k\}$ with $k \geq 3$. Then $s \leq 13$, and this bound can be attained.

Proof. By Theorem 3.12 we may assume that $s$ is odd. Then by Corollary 3.11 there exists a $\left(G, \frac{s+1}{2}\right)$-arc transitive graph of valency $k$. Thus by the result of Weiss [32], $\frac{s+1}{2} \leq 7$ and so $s \leq 13$. Moreover, examples of $(G, 7)-$ arc transitive graphs of valency at least three are well known (see [19]), and so by Corollary 3.11 locally $(G, 13)$-arc transitive graphs exist.

\section{EXAMPLES}

We now prove our assertions about the existence of locally $(G, s)$-arc transitive graphs with $s \geq 2$ for certain quasiprimitive types. We have already seen in Lemma 3.3 that there are examples of locally $(G, s)$-arc transitive graphs with $s \geq 2$ such that $G$ is quasiprimitive on both orbits of type HA, TW, AS or PA. Many more examples where $G$ is of type AS on both orbits can be found in [6, page 98]. From Theorem 1.2 the only remaining possible type when $G$ is quasiprimitive on both orbits is $\{\mathrm{SD}, \mathrm{PA}\}$, and the following example shows that such graphs do occur. 
Example 4.1. \{SD, PA\}: a family of locally 3-arc transitive graphs of valency $\{q, q+1\}$ with amalgam

$$
\left(\operatorname{PSL}(2, q) \times C_{2},\left(C_{2}^{2 f}: C_{q-1}\right): C_{2},\left(C_{2}^{f}: C_{q-1}\right) \times C_{2}\right) .
$$

Let $T=\operatorname{PSL}(2, q)$ for $q=2^{f} \geq 4$. Now $T$ has a 2 -transitive representation of degree $q+1$ and if $H$ is the stabiliser of a point in this representation, then $H \cong C_{2}^{f}: C_{q-1}$. The subgroup $H$ has a unique minimal normal subgroup $L \cong C_{2}^{f}$. Let $G=T$ wr $S_{2}$ and consider the graph $\Gamma=\operatorname{Cos}\left(G, G_{v}, G_{w}\right)$, where

$$
G_{v}=\{(t, t): t \in T\} \times S_{2}
$$

and

$$
G_{w}=\left\{\left(l_{1} h, l_{2} h\right): l_{i} \in L, h \in H\right\}: S_{2} .
$$

As $G_{v}$ is a maximal subgroup of $G, \Gamma$ is connected. The action of $G$ on $\Delta_{1}=\left[G: G_{v}\right]$ is primitive of type SD while the action of $G$ on $\Delta_{2}=\left[G: G_{w}\right]$ is quasiprimitive of type PA. Now $G_{v} \cap G_{w}=\{(h, h): h \in H\} \times S_{2}$. The action of $G_{v}$ on $\left[G_{v}: G_{v} \cap G_{w}\right]$ is then equivalent to the 2 -transitive representation of $T$ of degree $q+1$. The set $\{(l, 1): l \in L\}$ is a set of coset representatives for $G_{v} \cap G_{w}$ in $G_{w}$. For each $l \in L$, set $[l, 1]=\left(G_{v} \cap G_{w}\right)(l, 1)$. Now if $h \in H$, then $(h, h):[l, 1] \mapsto\left[l^{h}, 1\right]$. Then as $H$ induces the group $C_{q-1}$ as a group of automorphisms of $L$, it follows that $G_{v w}$ acts transitively on $\{[l, 1]: l \in L \backslash\{1\}\}$. Hence the permutation group induced by $G_{w}$ on the set of cosets $\left[G_{w}: G_{v} \cap G_{w}\right]$ is 2-transitive of degree $q$. Thus $\Gamma$ is locally $(G, 2)-\operatorname{arc}$ transitive with $G$ quasiprimitive of type $\{\mathrm{SD}, \mathrm{PA}\}$, and $\Gamma$ is biregular of valency $\{q+1, q\}$.

Let $v$ be the vertex in $\Delta_{1}$ corresponding to the coset $G_{v}$ and let $w$ be the vertex of $\Delta_{2}$ corresponding to the coset $G_{w}$. Let $w^{\prime} \in \Gamma(v) \backslash\{w\}$. Then

$$
G_{w^{\prime} v w} \cong\left\{(h, h): h \in C_{q-1}\right\} \times S_{2}
$$

which still acts transitively on $\Gamma(w) \backslash\{v\}$. Also, given $u \in \Gamma(w) \backslash\{v\}$,

$$
G_{u w v}=\{(l, l): l \in L\} \times S_{2}
$$

is still transitive on $\Gamma(v) \backslash\{w\}$. Hence $\Gamma$ is locally $(G, 3)-\operatorname{arc}$ transitive.

We now look at graphs where $G$ acts quasiprimitively on $\Delta_{1}$ but not quasiprimitively on $\Delta_{2}$. First we provide a family of examples for which $G$ acts primitively of type $\mathrm{HA}$ on $\Delta_{1}$.

Example 4.2. HA stars: a family of locally 3-arc transitive graphs of valency $\left\{q, \frac{q^{d}-1}{q-1}\right\}$ with amalgam

$$
\left(G L(d, q),[q]:\left([q]^{d-1}:\left(C_{q} \times \mathrm{GL}(d-1, q)\right)\right),[q]^{d-1}:\left(C_{q} \times \mathrm{GL}(d-1, q)\right)\right) .
$$

Define $\Gamma$ to be the bipartite graph whose vertices are all the points and lines of the affine space $\operatorname{AG}(d, q)$ and with adjacency given by incidence. There are $q^{d}$ points and $q^{d-1}\left(q^{d}-1\right) /(q-1)$ lines. Each point is adjacent to $\left(q^{d}-1\right) /(q-1)$ lines while each line is adjacent to $q$ points. Let $\operatorname{AGL}(d, q) \leqslant G \leqslant \operatorname{A\Gamma L}(d, q)$. Then $G$ is a group of automorphisms of $\Gamma$ with two orbits on the vertex set: the set $\Delta_{1}$ of points and the set $\Delta_{2}$ of lines. Given a point $p, G_{p}$ acts 2-transitively on the set of lines through $p$ while for any line $l, G_{l}$ acts 2 -transitively on the points of $l$. Thus $\Gamma$ is locally $(G, 2)-$ arc transitive and is biregular of valency $\left\{q, \frac{q^{d}-1}{q-1}\right\}$. In fact, it is not hard to show that $\Gamma$ is locally $(G, 3)-$ arc transitive. 
Now let $N$ be the group of translations of $G$. Then $N$ acts transitively on the set of points of $\operatorname{AG}(d, q)$ but has $\left(q^{d}-1\right) /(q-1)$ orbits on the set of lines. Each of these orbits is a set of parallel lines which partitions the set of points. Thus each point is incident to a unique line in each $N$-orbit and of course each line in an $N$-orbit is incident with $q$ points. Hence $\Gamma_{N}$ is the star $K_{1,\left(q^{d}-1\right) /(q-1)}$. As $N$ always has at least three orbits on $\Delta_{2}$, it follows from Lemma 5.6. which we will prove in the next section, that $\Gamma$ is never locally $(G, 4)-\operatorname{arc}$ transitive.

If we take $k$-dimensional affine subspaces, where $1<k<d-1$, instead of affine lines we get a $G$-locally primitive graph which is not locally $(G, 2)$-arc transitive. Its quotient graph modulo $N$ is again a star and its central vertex has valency

$$
\frac{\left(q^{d}-1\right)\left(q^{d-1}-1\right) \ldots\left(q^{d-k+1}-1\right)}{\left(q^{k}-1\right)\left(q^{k-1}-1\right) \ldots(q-1)} .
$$

The following family shows that examples also exist where $G$ is primitive of type $\mathrm{PA}$ on $\Delta_{1}$.

Example 4.3. PA stars: a family of locally 3-arc transitive graphs of valency $\{k, n\}$ with amalgam

$$
\left(S_{n-1} \text { wr } S_{k}, S_{n} \times\left(S_{n-1} \text { wr } S_{k-1}\right), S_{n-1}^{k}: S_{k-1}\right) .
$$

The Hamming graph $H(k, n)$ has vertices given by all the $k$-tuples with entries from a set $\Omega$ of size $n$. Let $k \geq 2, n \geq 5$, and let $\Delta_{1}$ be the set of vertices of $H(k, n)$. Also let $\Delta_{2}$ be the set of maximal cliques of $H(k, n)$. Define the graph $\Gamma$ to have vertex set $\Delta_{1} \cup \Delta_{2}$ and adjacency given by incidence. Then if $v=\left(\omega_{1}, \ldots, \omega_{k}\right) \in \Delta_{1}$, the maximal cliques in $\Delta_{2}$ adjacent to $v$ are the $k$ cliques $w=\left\{\left(\alpha, \omega_{2}, \ldots, \omega_{k}\right): \alpha \in \Omega\right\}$, $\left\{\left(\omega_{1}, \alpha, \omega_{3}, \ldots, \omega_{k}\right): \alpha \in \Omega\right\}, \ldots,\left\{\left(\omega_{1}, \ldots, \omega_{k-1}, \alpha\right): \alpha \in \Omega\right\}$.

Let $G=S_{n}$ wr $S_{k}$. Then the action of $G$ on $\Delta_{1}$ is primitive of type PA. Also $G_{v} \cong$ $S_{n-1}$ wr $S_{k}$ and $G_{v}^{\Gamma(v)} \cong S_{k}$ acting on $k$ points. Now $G_{w} \cong S_{n} \times\left(S_{n-1}\right.$ wr $\left.S_{k-1}\right)$ and $G_{w}^{\Gamma(w)} \cong S_{n}$ acting on $n$ points. Thus $\Gamma$ is locally $(G, 2)$-arc transitive and is biregular of valency $\{k, n\}$. Let $u=\left\{\left(\omega_{1}, \alpha, \omega_{3}, \ldots, \omega_{k}\right): \alpha \in \Omega\right\}$. Then $u \in \Gamma(v)$ and $G_{u v w} \cong\left(S_{n-1}\right)^{2} \times\left(S_{n-1}\right.$ wr $\left.S_{k-2}\right)$ which acts transitively on $\Gamma(w) \backslash\{v\}$. Also let $x=\left(\beta, \omega_{2}, \ldots, \omega_{k}\right)$ for some $\beta \in \Omega \backslash\left\{\omega_{1}\right\}$. Then $x \in \Gamma(w)$ and $G_{x w v} \cong S_{n-2} \times$ $\left(S_{n-1}\right.$ wr $\left.S_{k-1}\right)$ which acts transitively on $\Gamma(v) \backslash\{w\}$. Thus $\Gamma$ is locally $(G, 3)-\operatorname{arc}$ transitive. Let $N=S_{n}^{k} \triangleleft G$. Then $N$ is transitive on $\Delta_{1}$ but has $k$ orbits on $\Delta_{2}$, each orbit being the set of maximal cliques associated with a given coordinate. Thus $\Gamma_{N}=K_{1, k}$. Then by Lemma [5.6] $\Gamma$ is not locally $(G, 4)$-arc transitive when $k \geq 3$.

When $k=2$ the vertices of $\Delta_{1}$ have valency two. Also, given a vertex $\left\{\left(\alpha, \omega_{2}\right)\right.$ : $\alpha \in \Omega\}$ in $\Delta_{2}$ the vertices at distance two are all vertices of the form $\left\{\left(\omega_{1}, \alpha\right): \alpha \in\right.$ $\Omega\}$ for some $\omega_{1} \in \Omega$. Then the connected component $\widetilde{\Gamma}$ of the distance two graph of $\Gamma$ with $V \widetilde{\Gamma}=\Delta_{2}$ is $K_{n, n}$ and is $(G, 3)$-arc transitive. Thus by Theorem 3.10, $\Gamma$ is locally $(G, 5)-\operatorname{arc}$ transitive when $k=2$.

Note that for each $k \geq 2$ our last example provides us with a locally $(G, 3)$-arc transitive graph $\Gamma$ such that $G$ has a normal subgroup $N$ with $k$ orbits on $\Delta_{2}$ and $\Gamma_{N}=K_{1, k}$. Hence we have proved the following.

Lemma 4.4. Every star graph $K_{1, n}$ for $n \geq 2$ is the normal quotient of some locally 3-arc transitive graph and $K_{1,2}$ is the normal quotient of a locally 5-arc transitive graph. 
Examples where $G$ is quasiprimitive of type HS also occur.

Example 4.5. HS stars: a family of locally 3-arc transitive graphs of valency $\{q, q+1\}$ with amalgam

$$
\left(\operatorname{PSL}(2, q), C_{2}^{2 f}: C_{q-1}, C_{2}^{f}: C_{q-1}\right) .
$$

Let $\Gamma$ be the graph from Example 4.1 and let $G=T \times T$ where $T=\operatorname{PSL}(2, q)$. Then $\Gamma$ is locally $(G, 3)$-arc transitive such that $G$ acts primitively of type HS on $\Delta_{1}$ but has two normal subgroups $T \times 1$ and $1 \times T$ which are both intransitive on $\Delta_{2}$.

All examples where $G$ acts quasiprimitively of type $\mathrm{HS}$ on $\Delta_{1}$, yet has a normal subgroup which is intransitive on $\Delta_{2}$, will be determined in [12]. All such graphs are also of $\{\mathrm{SD}, \mathrm{PA}\}$ type. There are also examples for which $G$ is almost simple.

Example 4.6. AS star: a locally 3 -arc transitive graph of valency $\{3,8\}$ with amalgam

$$
\left(\left(C_{7}: C_{3}\right) \times S_{3}, \operatorname{PSL}(2,7) \times C_{2},\left(C_{7}: C_{3}\right) \times C_{2}\right) .
$$

Let $G=\operatorname{P\Gamma L}(3,4)$ which is an almost simple group with socle $T=\operatorname{PSL}(3,4)$. Let $\phi$ be a field automorphism of $T$ and consider $C_{T}(\phi) \cong \operatorname{PSL}(3,2) \cong \operatorname{PSL}(2,7)$. Let $R=N_{G}\left(C_{T}(\phi)\right)$. Then $R=\left\langle C_{T}(\phi), \phi\right\rangle \cong \operatorname{PSL}(2,7) \times C_{2} \leqslant \mathrm{P} \Sigma L(3,4)$. There are three conjugacy classes of subgroups of $T$ isomorphic to $C_{T}(\phi)$, and they are fused together by $G$. In fact $G / T \cong S_{3}$ permutes the three conjugacy classes naturally. Let $\delta$ be the element of $\operatorname{PGL}(3,4)$ corresponding to the matrix

$$
\left(\begin{array}{lll}
\xi & 0 & 0 \\
0 & 1 & 0 \\
0 & 0 & 1
\end{array}\right)
$$

where $\xi \in \mathrm{GF}(4)$ is of order three. Then $A=\langle\delta, \phi\rangle \cong S_{3}$ and $G=T: A$. Now let $L_{0}$ be the intersection of $C_{T}(\phi)$ and its two images under $A$. Then $L_{0}=C_{7}: C_{3}$ and its normaliser in $G$ is the maximal subgroup $L=\left(C_{7}: C_{3}\right) \times S_{3}$. (See [5].) Now $L \cap R=\left(C_{7}: C_{3}\right) \times C_{2}$. The action of $L$ on the set of cosets $[L: L \cap R]$ is equivalent to the action of $S_{3}$ on three points while the action of $R$ on the set of cosets $[R: L \cap R]$ is equivalent to the action of $\operatorname{PSL}(2,7)$ on 8 points. Both actions are 2 -transitive and so $\Gamma=\operatorname{Cos}(G, L, R)$ is a locally $(G, 2)$-arc transitive graph of valency $\{3,8\}$. One can deduce that $\Gamma$ is in fact locally $(G, 3)$-arc transitive. Furthermore, since $L$ is a maximal subgroup of $G, \Gamma$ is connected.

Further examples of locally $(G, 3)$-arc transitive graphs where $G$ is quasiprimitive of type AS on $\Delta_{1}$ but has a normal subgroup which is intransitive on $\Delta_{2}$ can be found in 13 . Finally we provide an example where $G$ is quasiprimitive of type TW on $\Delta_{1}$, so all types listed in Theorem 1.3 occur.

Example 4.7. TW star: a locally 2 -arc transitive graph of valency $\{6,16\}$ with amalgam $\left(A_{6}, C_{2}^{4}: A_{5}, A_{5}\right)$. Let $T=A_{5}, P=A_{6}, Q=A_{5}<P$, the stabiliser of the point 6 in the natural action and $\phi: Q \mapsto \operatorname{Inn}(T)$. Then $G=T \operatorname{twr}_{\phi} P$ has a normal subgroup $N \cong T^{6}$ and the action of $G$ on the set of cosets of $L=P$ is primitive of type TW. Let $\left\{z_{1}, \ldots, z_{5}, z_{6}=1\right\}$ be a transversal for $Q$ in $P$ and for each $i=1, \ldots, 6$, using the notation introduced in Section 2] let $T_{i}=\{f \in N$ : $f\left(z_{j}\right)=1$ for all $\left.j \neq i\right\}$. Then each $T_{i}$ is isomorphic to $T$ and $P$ acts on the set $\left\{T_{1}, \ldots, T_{6}\right\}$ such that $Q$ is the stabiliser of $T_{6}$. The subgroup $Q$ also normalises 
$T_{1} \times \cdots \times T_{5}$. For $i=1, \ldots, 5$, let $Q_{i}$ be the stabiliser in $Q$ of $T_{i}$ and let $V_{i}$ be the Klein 4-group of $Q_{i}$. Note that $V_{i} \triangleleft Q_{i}$. Let $R_{i}=\left\{f \in T_{i}: f\left(z_{i}\right) \in V_{i}\right\}$. Then for each $q \in Q$ we have $R_{i}^{q}=R_{i^{q}}$. Thus $M=R_{1} \times \cdots \times R_{5} \cong C_{2}^{10}$ is normalised by $Q$. Now $A_{5}$ does not have any irreducible representations of degree 10 over GF(2) so $Q$ normalises some proper subgroup of $M$. Calculations show that there exists $R_{0}$ in $M$ of order $2^{4}$ which is normalised by $Q$ and $Q$ acts irreducibly on $R_{0}$. Then let $R=R_{0}: Q \cong \operatorname{ASL}(2,4)$. Now $L \cap R=Q$ and the action of $L$ on the set of cosets $[L: L \cap R]$ is equivalent to the action of $A_{6}$ on six points. Furthermore, the action of $R$ on the set of cosets $[R: L \cap R]$ is equivalent to the 2-transitive action of $\operatorname{ASL}(2,4)$ on the $2^{4}$ points of the affine plane $\operatorname{AG}(2,4)$. Thus the graph $\Gamma=\operatorname{Cos}(G, L, R)$ is locally $(G, 2)-\operatorname{arc}$ transitive and is biregular of valency $\{6,16\}$. Now $G$ acts primitively on $\Delta_{1}=[G: L]$ but $N$ has six orbits on $\Delta_{2}=[G: R]$. Hence the quotient graph $\Gamma_{N}$ is the star $K_{1,6}$. The graph $\Gamma$ is not locally $(G, 3)-$ arc transitive as given, $v=L \in \Delta_{1}, w=R \in \Delta_{2}$ and $w^{\prime} \in \Gamma(v) \backslash\{w\}$, we have $G_{w^{\prime} v w}=A_{4}$ and there are 15 vertices in $\Gamma(w) \backslash\{v\}$.

\section{Normal QUOTIEnts}

In this section we investigate the normal quotients of a $G$-edge transitive bipartite graph $\Gamma$ and in particular we prove Theorem 1.1 We begin by showing that taking quotients with respect to the orbits of a normal subgroup which is intransitive on both $G$-orbits, preserves local primitivity and local $s$-arc transitivity.

Lemma 5.1. Let $\Gamma$ be a connected $G$-locally primitive bipartite graph with $G$-orbits $\Delta_{1}$ and $\Delta_{2}$ on $V \Gamma$ and each $\left|\Delta_{i}\right|>1$. Suppose that there exists $N \triangleleft G$ such that $N$ is intransitive on $\Delta_{1}$ and on $\Delta_{2}$. Then

(1) $\Gamma$ is a cover of $\Gamma_{N}$.

(2) $N$ acts semiregularly on $V \Gamma$ and $G^{V \Gamma_{N}} \cong G / N$.

(3) $\Gamma_{N}$ is $(G / N)$-locally primitive. Furthermore, if $\Gamma$ is locally $(G, s)$-arc transitive, then $\Gamma_{N}$ is locally $(G / N, s)$-arc transitive.

Proof. Let $v \in \Delta_{1}$ and let $B=v^{N}$. Choose $u \in \Gamma(v) \subseteq \Delta_{2}$ and set $C=u^{N}$. Then $C$ is a block of imprimitivity for the action of $G$ on $\Delta_{2}$, and hence $C \cap \Gamma(v)$ is a block of imprimitivity for the action of $G_{v}$ on $\Gamma(v)$. As $N_{v} \triangleleft G_{v}$ and $G_{v}$ acts primitively on $\Gamma(v)$, it follows that either $\Gamma(v) \subseteq C$ or $|\Gamma(v) \cap C|=1$.

Suppose that $\Gamma(v) \subseteq C$. Then for every vertex $w \in \Delta_{1}$, the set $\Gamma(w)$ is contained in some $N$-orbit. Thus if $B^{\prime}$ is an $N$-orbit on $\Delta_{1}$ containing a vertex adjacent to a vertex in $C$, then $\Gamma\left(B^{\prime}\right) \subseteq C$. It follows from the connectedness of $\Gamma$ that $V \Gamma=C \cup \Gamma(C)$, contradicting the intransitivity of $N$ on $\Delta_{2}$. Thus $|\Gamma(v) \cap C|=1$. Similarly $|\Gamma(u) \cap B|=1$ and so $\Gamma$ is a cover of $\Gamma_{N}$.

Let $K$ be the kernel of the action of $G$ on the set of $N$-orbits on $V \Gamma$ and let $v \in V \Gamma$. Now $K_{v}$ fixes each $N$-orbit setwise and since distinct vertices of $\Gamma(v)$ lie in distinct $N$-orbits, we have that $K_{v}$ acts trivially on $\Gamma(v)$. Since $\Gamma$ is connected it follows that $K_{v}$ fixes all the vertices of $\Gamma$ and hence $K_{v}=1$. Since this is true for all $v, K$ acts semiregularly on $V \Gamma$ and hence so does $N$. Furthermore, as $N \leqslant K$ and acts transitively on the orbits of $K$, we see that $K=N$. Thus $G^{V \Gamma_{N}} \cong G / N$ and so $G / N \leqslant \operatorname{Aut}\left(\Gamma_{N}\right)$.

For a vertex $v$ in the $N$-orbit $B$, the group $N G_{v}$ fixes $B$, contains $G_{v}$ and is transitive on $B$. Hence $G_{B}=N G_{v}$. Then as $N$ is the kernel of the action of $G$ on $V \Gamma_{N}$ and as each block in $\Gamma_{N}(B)$ contains exactly one vertex of $\Gamma(v)$, we have 
that $G_{B}^{\Gamma_{N}(B)}$ is permutationally isomorphic to $G_{v}^{\Gamma(v)}$ and so is primitive. Thus $\Gamma_{N}$ is $(G / N)$-locally primitive.

Let $\left(B, B_{1}, \ldots, B_{s}\right)$ and $\left(B, C_{1}, \ldots, C_{s}\right)$ be $s$-arcs in $\Gamma_{N}$. Choose $v \in B$. Then there exist unique $v_{i} \in B_{i}$ and $u_{i} \in C_{i}$ such that $\left(v, v_{1}, \ldots, v_{s}\right)$ and $\left(v, u_{1}, \ldots, u_{s}\right)$ are $s$-arcs in $\Gamma$. If $\Gamma$ is locally $(G, s)$-arc transitive, then there exists $g \in G_{v}$ mapping one to the other. As the orbits of $N$ form a system of imprimitivity for $G$, it follows that $g \in G_{B}$ and maps the $s-\operatorname{arc}\left(B, B_{1}, \ldots, B_{s}\right)$ to $\left(B, C_{1}, \ldots, C_{s}\right)$. Thus $\Gamma_{N}$ is locally $(G / N, s)$-arc transitive.

Note that if $N$ is maximal in $G$ subject to being intransitive on both orbits $\Delta_{1}$ and $\Delta_{2}$, then each normal subgroup of $G$ containing $N$ is transitive on at least one $G$-orbit.

We now consider the question of faithfulness.

Lemma 5.2. Let $\Gamma$ be a connected $G$-locally primitive bipartite graph with $G$-orbits $\Delta_{1}$ and $\Delta_{2}$ on $V \Gamma$ of sizes $n$ and $n^{\prime}$, respectively. Then either $\Gamma \cong K_{n, n^{\prime}}$ or $G$ is faithful on both $\Delta_{1}$ and $\Delta_{2}$.

Proof. If either of $n$ or $n^{\prime}$ is 1 , then $\Gamma=K_{n, n^{\prime}}$ so assume that both $n$ and $n^{\prime}$ are at least 2. Let $K_{1}$ and $K_{2}$ be the kernels of $G$ on $\Delta_{1}$ and $\Delta_{2}$, respectively. Since $G$ acts faithfully on $V \Gamma$, we know that $K_{1} \cap K_{2}=1$.

Suppose that $K_{1} \neq 1$ and note that $K_{1}$ acts faithfully on $\Delta_{2}$. Let $B$ be a nontrivial orbit of $K_{1}$ on $\Delta_{2}$ and $u$ a vertex in $B$. Let $v$ be a vertex in $\Delta_{1}$ adjacent to $u$. Since $K_{1}$ fixes $v$, it follows that $v$ is adjacent to every vertex in $B$. Furthermore, as $K_{1} \triangleleft G_{v}$, the orbits of $K_{1}$ on $\Gamma(v)$ are blocks of imprimitivity for the action of $G_{v}$. Then as $\Gamma$ is $G$-locally primitive, $\Gamma(v)=B$. As this holds for every $v$ adjacent to a vertex of $B$, it follows from the connectedness of $\Gamma$ that $\Gamma \cong K_{n, n^{\prime}}$. The same holds if $K_{2} \neq 1$.

Note that if either $n$ or $n^{\prime}$ is at least three and neither is equal to one, then $K_{n, n^{\prime}}$ is locally $3-\operatorname{arc}$ transitive but not locally 4 -arc transitive as there are $4-$ $\operatorname{arcs}\left(v_{0}, v_{1}, v_{2}, v_{3}, v_{4}\right)$ for which $v_{4}=v_{0}$ and some for which $v_{4} \neq v_{0}$. The graph $K_{2,2}$ is a cycle of length four and is locally $(G, s)$-arc transitive for all $s \geq 1$ with $G=S_{2} \times S_{2}$. On the other hand, the graphs $K_{1, n}$ for $n \geq 2$ are only locally $2-$ arc transitive as they do not contain any 3 -arcs.

We prove the following lemma regarding edge transitive graphs with a vertex of valency one.

Lemma 5.3. Let $\Gamma$ be a connected $G$-edge transitive but not $G$-vertex transitive graph such that $|\Gamma(u)|=1$ for some vertex $u$. Then $\Gamma$ is a star $K_{1, k}$, and if $G$ acts faithfully on both $G$-orbits on vertices, then $k=1, \Gamma=K_{2}$ and $G=1$.

Proof. Let $\Delta_{1}$ and $\Delta_{2}$ be the $G$-orbits on $V \Gamma$. Without loss of generality we may suppose that $u \in \Delta_{1}$. Since $u$ is joined by an edge to only one vertex of $\Delta_{2}$ and $\Gamma$ is connected, $\Gamma$ is a star $K_{1, k}$. If $G$ is faithful on $\Delta_{2}$, then $G=1$ and hence $\left|\Delta_{1}\right|=1$. Thus $\Gamma=K_{1,1}=K_{2}$.

Now assuming that $G$ acts faithfully on both of its orbits we investigate the case where every nontrivial normal subgroup of $G$ is transitive on at least one $G$-orbit.

Lemma 5.4. Let $\Gamma$ be a finite connected graph such that $G$ has two orbits $\Delta_{1}$ and $\Delta_{2}$ on vertices and $G$ acts faithfully on both orbits. Suppose that every nontrivial 
normal subgroup $N$ of $G$ is transitive on at least one of the $\Delta_{i}$. Then $G$ acts quasiprimitively on at least one of its orbits.

Proof. Suppose that $G$ is not quasiprimitive on either of the $\Delta_{i}$. Then for each $i \in\{1,2\}$ there exists $N_{i} \triangleleft G$ such that $N_{i}$ is intransitive on $\Delta_{i}$ and transitive on $\Delta_{3-i}$. Now $N_{1} \cap N_{2} \triangleleft G$ and so if nontrivial would be transitive on at least one $\Delta_{i}$. But then $N_{1}$ and $N_{2}$ would both be transitive on the same set, a contradiction. Thus $N_{1} \cap N_{2}=1$ and hence $N_{1} \times N_{2} \triangleleft G$.

As each $N_{i}$ is transitive on $\Delta_{3-i}$, it follows that $C_{\operatorname{Sym}\left(\Delta_{3-i}\right)}\left(N_{i}\right)$ is semiregular. Thus each $N_{i}$ is semiregular on $\Delta_{i}$ and transitive on $\Delta_{3-i}$. Therefore $\left|N_{1}\right|$ divides $\left|\Delta_{1}\right|$ and $\left|\Delta_{2}\right|$ divides $\left|N_{1}\right|$, so $\left|\Delta_{2}\right|$ divides $\left|\Delta_{1}\right|$. Similarly $\left|\Delta_{1}\right|$ divides $\left|N_{2}\right|$ and $\left|N_{2}\right|$ divides $\left|\Delta_{2}\right|$ implying that $\left|\Delta_{1}\right|$ divides $\left|\Delta_{2}\right|$. Thus $\left|\Delta_{1}\right|=\left|\Delta_{2}\right|$. Furthermore, $\left|N_{1}\right|=\left|\Delta_{1}\right|=\left|N_{2}\right|$, contradicting $N_{1}$ being intransitive on $\Delta_{1}$. Thus $G$ must be quasiprimitive on at least one of the $\Delta_{i}$.

Note that this is the only lemma necessary for the proof of Theorem 1.1 whose proof does not extend to the case of infinite locally $s$-arc transitive graphs.

We now analyse the structure of $\Gamma$ in the case where $G$ has a normal subgroup which is transitive on one $G$-orbit yet intransitive on the other. A proof of Lemma 5.5 will complete the proof of Theorem 1.1 .

Lemma 5.5. Let $\Gamma$ be a connected $G$-locally primitive bipartite graph with $G$-orbits $\Delta_{1}$ and $\Delta_{2}$ on $V \Gamma$. Suppose there exists $N \triangleleft G$ such that $N$ is transitive on $\Delta_{1}$ but intransitive on $\Delta_{2}$. Then $\Gamma_{N}$ is a star whose central vertex has valency the number of orbits of $N$ on $\Delta_{2}$. Furthermore, for each vertex $v \in \Delta_{1}$ and $N$-orbit $B$ in $\Delta_{2}$, $|B \cap \Gamma(v)|=1$ and the vertex stabiliser $N_{v}$ acts trivially on $\Gamma(v)$.

Proof. Choose $u \in \Delta_{2}$ and $v \in \Delta_{1}$ such that $v \sim u$. Let $B=u^{N}$. For each $w \in B$, we have that $w=u^{g}$ for some $g \in N$ and $v^{g} \in \Gamma\left(u^{g}\right)=\Gamma(w)$, that is, each vertex of $B$ is adjacent to some vertex in $\Delta_{1}$. Conversely, as $N$ acts transitively on $\Delta_{1}$ each vertex of $\Delta_{1}$ is adjacent to some vertex in $B$. Thus $\Gamma_{N}$ is a star whose central vertex has valency the number of orbits of $N$ on $\Delta_{2}$.

Now the set $\Gamma(v) \cap B$ is an orbit of $N_{v}$ on $\Gamma(v)$ and is hence a block for $G_{v}$. If $\Gamma(v) \subseteq B$, then $\Gamma\left(v^{\prime}\right) \subseteq B$ for all $v^{\prime} \in \Delta_{1}$ since $B$ and $\Delta_{1}$ are $N$-orbits, and this contradicts the connectivity of $\Gamma$. Hence $|B \cap \Gamma(v)|=1$ and so $N_{v}$ acts trivially on $\Gamma(v)$.

Finally, in this section we state a lemma from [17] and give a short proof.

Lemma 5.6. Let $\Gamma$ be a locally $(G, s)$-arc transitive graph such that all vertices have valency at least two and $G$ has a normal subgroup $N$ which is transitive on $\Delta_{1}$ but has at least three orbits on $\Delta_{2}$. Then $s \leq 3$.

Proof. Let $B_{1}, B_{2}$ and $B_{3}$ be three orbits of $N$ on $\Delta_{2}$. Choose $v_{0} \in B_{1}$ and let $v_{1} \in \Gamma\left(v_{0}\right)$. Then by Lemma 5.5, $v_{1}$ is adjacent to a unique vertex $v_{2}$ in $B_{2}$. Let $v_{3} \in \Gamma\left(v_{2}\right) \backslash\left\{v_{2}\right\}$. Then $\left(v_{0}, v_{1}, v_{2}, v_{3}\right)$ is a 3 -arc in $\Gamma$. Now by Lemma 5.5, there exist $u, w \in \Gamma\left(v_{3}\right)$ such that $u \in B_{1}$ and $w \in B_{3}$. Then $\left(v_{0}, v_{1}, v_{2}, v_{3}, u\right)$ and $\left(v_{0}, v_{1}, v_{2}, v_{3}, w\right)$ are 4 -arcs in $\Gamma$ for which there does not exist an element $g \in G_{v_{0}}$ mapping one to the other, since such a $g$ would fix $B_{1}$ and so cannot map $u$ to $w$. Hence $\Gamma$ is not locally $(G, 4)$-arc transitive. 


\section{QuASIPRIMITIVE ON BOTH ORBITS}

In this section we analyse the case where $G$ acts faithfully and quasiprimitively on both of its orbits. In particular we prove Theorem[1.2.

We say that $\Gamma$ is $G$-locally quasiprimitive if $G_{v}^{\Gamma(v)}$ is a quasiprimitive permutation group for every vertex $v$. We begin with two very useful lemmas.

Lemma 6.1. Let $\Gamma$ be a connected $G$-locally quasiprimitive graph. Suppose that $\Gamma$ is bipartite and the orbits of $G$ are the bipartite halves $\Delta_{1}$ and $\Delta_{2}$. Suppose also that $G$ acts faithfully and quasiprimitively on $\Delta_{1}$ and on $\Delta_{2}$. If $N \triangleleft G$, then $N^{\Delta_{1}}$ is regular if and only if $N^{\Delta_{2}}$ is regular.

Proof. If there exists a vertex with valency one, then by Lemma [5.3, $\Gamma=K_{2}$ and the result is trivially true. So we assume $|\Gamma(v)| \geq 2$ for all $v \in V \Gamma$. Suppose that $N^{\Delta_{1}}$ is regular and $N^{\Delta_{2}}$ is not regular. Then for all $v \in \Delta_{1}, N_{v}=1$, while there exists $u \in \Delta_{2}$ such that $N_{u} \neq 1$. This implies that $N_{u}$ acts nontrivially on $\Gamma(u)$ and so

$$
1 \neq N_{u}^{\Gamma(u)} \triangleleft G_{u}^{\Gamma(u)} .
$$

As $G_{u}^{\Gamma(u)}$ is a quasiprimitive permutation group, $N_{u}$ acts transitively on $\Gamma(u)$. Since $G$ is quasiprimitive and faithful on $\Delta_{2}, N$ is transitive on $\Delta_{2}$ and so for all $w \in \Delta_{2}$, $N_{w}$ acts transitively on $\Gamma(w)$. This implies that $N$ is transitive on the set of edges of $\Gamma$. Thus $N_{v}$ acts transitively on $\Gamma(v)$ which contradicts $N_{v}=1$ as $v$ has at least two neighbours.

Lemma 6.2. Let $\Gamma$ and $G$ be as in Lemma 6.1 and let $N \triangleleft G$. If $N$ is not regular on $\Delta_{1}$, then $N_{v}^{\Gamma(v)}$ is transitive for all $v \in V \Gamma$.

Proof. If $\Gamma$ has a vertex of valency one, then by Lemma 5.3, $\Gamma=K_{2}$ and $G=1$ so no such $N$ exists. Thus all vertices have valency at least two. By Lemma 6.1, $N$ is not regular on $\Delta_{2}$ either, and so for all $v \in V \Gamma, N_{v} \neq 1$. Suppose that there exists $v \in V \Gamma$ such that $N_{v}^{\Gamma(v)}=1$. Then as $\Gamma$ is connected and $G$ acts faithfully on $V \Gamma$, there exists a path $\left(v_{0}, v_{1}, \ldots, v_{r}\right)$ in $\Gamma$ with $v_{0}=v$ such that $N_{v}$ fixes $v_{0}, \ldots, v_{r-1}$ but not $v_{r}$. Now $N_{v} \leqslant N_{v_{r-1}}$ and $N_{v}$ moves $v_{r} \in \Gamma\left(v_{r-1}\right)$ so

$$
1 \neq N_{v_{r-1}}^{\Gamma\left(v_{r-1}\right)} \triangleleft G_{v_{r-1}}^{\Gamma\left(v_{r-1}\right)} .
$$

Now $G_{v_{r-1}}^{\Gamma\left(v_{r-1}\right)}$ is quasiprimitive, so $N_{v_{r-1}}^{\Gamma\left(v_{r-1}\right)}$ is transitive. The vertex $v_{r-1}$ is in $\Delta_{1}$ or in $\Delta_{2}$ and so as $N$ is transitive on each of the sets $\Delta_{1}$ and $\Delta_{2}$, we have that $N$ is transitive on the edges of $\Gamma$. This contradicts $N_{v}^{\Gamma(v)}=1$ as $v$ has at least two neighbours. Thus $N_{v}^{\Gamma(v)} \neq 1$ and arguing as above, $N_{v}^{\Gamma(v)}$ is transitive.

We are now in a position to determine the possible quasiprimitive types for $G$.

Proposition 6.3. Let $\Gamma$ be a finite connected $G$-locally primitive graph with $G$ orbits $\Delta_{1}$ and $\Delta_{2}$ on vertices. Suppose that $G$ acts faithfully and quasiprimitively on $\Delta_{1}$ and on $\Delta_{2}$ of type $\{X, Y\}$. Then either $X=Y$, or $\{X, Y\}=\{\mathrm{SD}, \mathrm{PA}\}$ or $\{\mathrm{CD}, \mathrm{PA}\}$.

Proof. Note that $G^{\Delta_{1}} \cong G^{\Delta_{2}} \cong G$. So if $G$ is of type HA, AS, HS or HC on one of the $G$-orbits, then it must have the same type on the other $G$-orbit, as such types are determined by the abstract structure and number of minimal normal subgroups of $G$. 
If $G$ is of type TW on one $G$-orbit, then $G$ has a unique minimal normal subgroup $N$ isomorphic to $T^{k}$ for some finite nonabelian simple group $T$ and $N$ is regular on that orbit. Lemma 6.1 implies that $N$ also acts regularly on the other $G-$ orbit and so $G$ must be of type TW on both orbits. Thus we may assume that $\{X, Y\} \subseteq\{\mathrm{SD}, \mathrm{CD}, \mathrm{PA}\}$, and so the only thing that remains to be proved is that $\{X, Y\} \neq\{\mathrm{SD}, \mathrm{CD}\}$.

Suppose to the contrary that $G$ is of type $\{\mathrm{SD}, \mathrm{CD}\}$ and without loss of generality assume that $G$ is quasiprimitive of type SD on $\Delta_{1}$ and quasiprimitive of type CD on $\Delta_{2}$. Let $N$ be the unique minimal normal subgroup of $G$. Then $N \cong T^{k}$ for some finite nonabelian simple group $T$. Since $G$ acts faithfully on $\Delta_{1}$, we may assume that $N<G<N$. $\left(\operatorname{Out}(T) \times S_{k}\right)<\operatorname{Aut}(T)$ wr $S_{k}$ and for some $v \in \Delta_{1}$, we have $N_{v}=\{(t, \ldots, t): t \in T\}$. Hence a typical element of $G$ is of the form $\left(t_{1}, \ldots, t_{k}\right)(\sigma, \ldots, \sigma) \pi$, where the $t_{i} \in T, \sigma \in \operatorname{Aut}(T)$ and $\pi \in S_{k}$. Let $w \in \Gamma(v)$. Then as $G$ is quasiprimitive on $\Delta_{2}$ of type CD, $N_{w}=D_{1} \times \cdots \times D_{l}$ where each $D_{i}$ is a full diagonal subgroup of $T^{m}$ where $k=m l, l \geq 2$. Thus $D_{1}=\left\{t, t^{\varphi_{1_{2}}}, \ldots, t^{\varphi_{1_{m}}}\right)$ : $t \in T\}$ where $\varphi_{1_{2}}, \ldots, \varphi_{1_{m}} \in \operatorname{Aut}(T)$ and the other $D_{i}$ are conjugates of $D_{1}$ under elements of $G$. So we may assume that $D_{i}=\left\{\left(t, t^{\varphi_{i_{2}}}, \ldots, t^{\varphi_{i_{m}}}\right): t \in T\right\}$ for some $\varphi_{i_{j}} \in \operatorname{Aut}(T)$, and $G \leqslant T^{k}$. $\left(\operatorname{Out}(T) \times\left(S_{m} \operatorname{wr} S_{l}\right)\right)$. Thus every element $g \in G_{v}$ is of the form $(\sigma, \ldots, \sigma) \tau$ for $\sigma \in \operatorname{Aut}(T)$ and $\tau \in S_{m}$ wr $S_{l}$. Now $N_{v} \cap N_{w}=\{(t, \ldots, t)$ : $t \in C\} \triangleleft G_{v} \cap G_{w}$ where $C$ is the centraliser in $T$ of all the $\varphi_{i_{j}}$. If all the $\varphi_{i_{j}}=1$, then $N_{v} \leq N_{w}$ and so as $G_{v}^{\Gamma(v)}$ is primitive it follows that $N_{v}^{\Gamma(v)}=1$. Then Lemma 6.2 implies that $N_{v}=1$, which contradicts $\left|N_{v}\right|=|T|$. Thus at least one of the $\varphi_{i_{j}}$ is nontrivial and $C \neq T$. Let $g \in G_{v} \cap G_{w}$. Then $g=(\sigma, \ldots, \sigma) \tau$ where $\sigma$ normalises $C$ and so $g$ normalises the subgroup $A=C_{1} \times \cdots \times C_{k}$ where each $C_{i}=\{(c, \ldots, c): c \in C\}<D_{i}$. Let $H=\left\langle G_{v} \cap G_{w}, A\right\rangle$. Then $H=A\left(G_{v} \cap G_{w}\right)$ and $H \cap N_{w}=A \neq N_{v} \cap N_{w}$. Thus $G_{v} \cap G_{w}<H<G_{w}$, contradicting $G_{v} \cap G_{w}$ being maximal in $G_{w}$. Hence $\Gamma$ cannot be of type $\{\mathrm{SD}, \mathrm{CD}\}$.

In the locally $(G, s)$-arc transitive case we can prove even more.

Lemma 6.4. There are no connected locally $(G, s)$-arc transitive graphs with $s \geq 2$ such that $G$ acts faithfully and quasiprimitively on both orbits and of type $H C$ or $C D$ on one.

Proof. Suppose such a graph $\Gamma$ exists, and that $G$ is of type $\mathrm{HC}$ or CD on $\Delta_{1}$. Then $G$ has socle $N=T_{1} \times \cdots \times T_{k} \cong T^{k}$ for some finite nonabelian simple group $T$ and $k \geq 2$. Let $v \in \Delta_{1}$. Then there exists an integer $l \geq 2$ dividing $k$ such that without loss of generality, $N_{v}=D_{1} \times \cdots \times D_{l}$ where each $D_{i}$ is a full diagonal subgroup of $T_{k(i-1) / l+1} \times \cdots \times T_{k i / l}$. If $G$ is of type $\mathrm{HC}$, then $l=k / 2$ and for either $\mathrm{HC}$ or CD type, $G_{v}$ permutes $D_{1}, \ldots, D_{l}$ transitively by conjugation. Now as $N$ does not act regularly on $\Delta_{1}$, Lemma 6.2 implies that

$$
1 \neq N_{v}^{\Gamma(v)} \triangleleft G_{v}^{\Gamma(v)} .
$$

Then by a theorem of Burnside [3, Theorem 4.3] we see that $N_{v}^{\Gamma(v)} \cong T$. If $\operatorname{ker}\left(N_{v}^{\Gamma(v)}\right)$ denotes the kernel of the action of $N_{v}$ on $\Gamma(v)$, then $\operatorname{ker}\left(N_{v}^{\Gamma(v)}\right) \cong T^{l-1}$. Also, since $\operatorname{ker}\left(N_{v}^{\Gamma(v)}\right) \triangleleft N_{v}$, it follows that $\operatorname{ker}\left(N_{v}^{\Gamma(v)}\right)$ is a product of $l-1$ of the $D_{i}$. However, as $G_{v}$ acts transitively by conjugation on $\left\{D_{1}, \ldots, D_{l}\right\}$ and $G_{v}$ normalises $\operatorname{ker}\left(N_{v}^{\Gamma(v)}\right)$, this is a contradiction. 
We saw in Lemma 3.3 that there are locally $(G, s)$-arc transitive graphs with $G$ of type HA, AS, TW and PA on both $G$-orbits and by Lemma 6.4 there are no such graphs of type $\{X, Y\}$ with $\mathrm{HC}$ or $\mathrm{CD}$ in $\{X, Y\}$. Therefore by Proposition 6.3 if such a graph has type $\{X, Y\}$ with $X \neq Y$, then $\{X, Y\}=\{\mathrm{SD}, \mathrm{PA}\}$. Example 4.1 provides an example of such a graph. We now complete the proof of Theorem 1.2 by showing that locally $(G, s)$-arc transitive graphs of type HS or SD do not exist. The argument is similar to that in [22].

Lemma 6.5. There are no connected locally $(G, s)$-arc transitive graphs with $G$ acting faithfully on both orbits and quasiprimitive of type $H S$ or $S D$.

Proof. Suppose that such a graph $\Gamma$ does exist. Let $X$ be the socle of $G$. Then $X \cong T^{k}$ for some finite nonabelian simple group $T$ and $k \geq 2$. We can identify $\Delta_{1}$ and $\Delta_{2}$ with the elements of $T^{k-1}$ such that the action of $X$ on $T^{k-1}$ is given by

$$
\left(t_{1}, \ldots, t_{k}\right):\left(a_{1}, \ldots, a_{k-1}\right) \mapsto\left(t_{k}^{-1} a_{1} t_{1}, \ldots, t_{k}^{-1} a_{k-1} t_{k-1}\right) .
$$

Now $T \cong X_{v} \triangleleft G_{v}$. Also, by Lemma 6.2 $X_{v}^{\Gamma(v)} \neq 1$ and so is isomorphic to $T$. Furthermore, since $G_{v}$ acts 2-transitively on $\Gamma(v)$, a theorem of Burnside (see [3] Theorem 4.3]) implies that the action of $X_{v}$ on $\Gamma(v)$ is primitive.

Let $v=\left(1_{T}, \ldots, 1_{T}\right) \in \Delta_{1}$. Then $X_{v}=\{(t, \ldots, t): t \in T\}$. Also for $u \in \Gamma(v)$ we have that $X_{u}=\left\{\left(t, t^{\varphi_{2}}, \ldots, t^{\varphi_{k}}\right): t \in T\right\}$ for some $\varphi_{2}, \ldots, \varphi_{k} \in \operatorname{Aut}(T)$. Now $X_{v, u}=\left\{(t, \ldots, t): t \in C_{T}\left(\varphi_{i}\right)\right.$ for all $\left.i\right\}$. As $X_{v} \neq X_{v, u}$, at least one $\varphi_{i}$ is not the identity. Furthermore, as $X_{v, u}$ is a maximal subgroup of $X_{v}$, we have that $X_{v, u} \cong C_{T}\left(\varphi_{i}\right)$. However, $G_{v}$ is a 2 -transitive almost simple group on $\Gamma(v)$ and no such group exists where the point stabiliser of the socle is a centraliser of a (possibly outer) automorphism. (See [3, Section 7.4].) Thus no such graph $\Gamma$ exists.

It remains to provide examples of $G$-locally primitive graphs to prove our assertions about the existence of such graphs for the remaining possible quasiprimitive types for $G$-locally primitive graphs which are not possible for locally $(G, s)$-arc transitive graphs, namely the types $\{\mathrm{CD}, \mathrm{PA}\}$ and the types $X=Y \in\{\mathrm{HS}, \mathrm{SD}, \mathrm{HC}$, $\mathrm{CD}\}$. The constructions given in Example 6.6 below therefore complete the proof of Theorem 1.2 .

Example 6.6. (1) (Type $\{\mathrm{CD}, \mathrm{PA}\}$ of valency $\left\{n^{2},\left|A_{n-1}\right|^{2}\right\}$ ) Let $T=A_{n}$ for $n \geq 6$ and let $G=T^{4}:\left(S_{2} \operatorname{wr} S_{2}\right)=T \operatorname{wr}\left(S_{2} \operatorname{wr} S_{2}\right)$. The action of $G$ on the set of right cosets of the subgroup

$$
G_{v}=\{(t, t, s, s): t, s \in T\}:\left(S_{2} \operatorname{wr} S_{2}\right)
$$

is primitive of type CD. The group $G$ also acts on the set of right cosets of $G_{w}=$ $A_{n-1}^{4}:\left(S_{2}\right.$ wr $\left.S_{2}\right)$ with a primitive action of type PA. Consider the graph $\Gamma=$ $\operatorname{Cos}\left(G, G_{v}, G_{w}\right)$. Here $G$ acts primitively on its two orbits on vertices with type $\{\mathrm{CD}, \mathrm{PA}\}$. Now

$$
G_{v} \cap G_{w}=\left\{(t, t, s, s): t, s \in A_{n-1}\right\}:\left(S_{2} \mathrm{wr} S_{2}\right)
$$

which is a maximal subgroup of both $G_{v}$ and $G_{w}$. Actually $G_{v}$ is primitive on $\Gamma(v)$ of type $\mathrm{PA}$, and $G_{w}$ is primitive on $\Gamma(w)$ of type CD. Thus $\Gamma$ is a $G$-locally primitive connected graph which is biregular of valency $\left\{n^{2},\left|A_{n-1}\right|^{2}\right\}$.

(2) (Type HS and SD of valency $\left|T: C_{T}(\sigma)\right|$ ) Let $T$ be a finite nonabelian simple group with automorphism $\sigma$ such that $C_{T}(\sigma)$ is a maximal subgroup of $T$. For example, $T=A_{n}$ and $\sigma$ is the automorphism induced by conjugation by $(1,2)$. 
Let $G=T \times T, G_{v}=\{(t, t): t \in T\}$ and $G_{w}=\left\{\left(t, t^{\sigma}\right): t \in T\right\}$. Consider the graph $\Gamma=\operatorname{Cos}\left(G, G_{v}, G_{w}\right)$. Then the actions of $G$ on $\Delta_{1}=\left[G: G_{v}\right]$ and $\Delta_{2}=\left[G: G_{w}\right]$ are primitive of type HS. Also $\left\langle G_{v}, G_{w}\right\rangle=G$, so $\Gamma$ is connected. Now $G_{v} \cap G_{w}=\left\{(t, t): t \in C_{T}(\sigma)\right\}$ which is a maximal subgroup of both $G_{v}$ and $G_{w}$. Hence $\Gamma$ is $G$-locally primitive with $G$ of type HS and of valency $\left|T: C_{T}(\sigma)\right|$.

Suppose that $\sigma$ has order two and let $\bar{G}=G: S_{2}$. Then $\bar{G} \leqslant \operatorname{Aut}(\Gamma)$ and acts primitively on both $\Delta_{1}$ and $\Delta_{2}$ with type SD. Furthermore, $\Gamma$ is also $\bar{G}$-locally primitive.

(3) (Type HC and CD of valency $\left|T: C_{T}(\sigma)\right|^{2}$ ) Take $T$ to be a finite nonabelian simple group with an automorphism $\sigma$ of order two such that $C_{T}(\sigma)$ is a maximal subgroup of $T$. Let $G=\left(T^{2} \times T^{2}\right): S_{2}$ where $S_{2}$ is generated by the element $(1,2)(3,4)$. Then $G$ has two minimal normal subgroups each isomorphic to $T^{2}$. In addition, let

$$
G_{v}=\{(t, s, t, s): t, s \in T\}: S_{2}
$$

and

$$
G_{w}=\left\{\left(t, s, t^{\sigma}, s^{\sigma}\right): t, s \in T\right\}: S_{2} .
$$

Consider the graph $\Gamma=\operatorname{Cos}\left(G, G_{v}, G_{w}\right)$. Then as $\left\langle G_{v}, G_{w}\right\rangle=G, \Gamma$ is connected and the actions of $G$ on $\Delta_{1}$ and $\Delta_{2}$ are quasiprimitive of type HC. Now

$$
G_{v} \cap G_{w}=\left\{(t, s, t, s): t, s \in C_{T}(\sigma)\right\}: S_{2}
$$

which is a maximal subgroup of both $G_{v}$ and $G_{w}$. In fact $G_{v}$ and $G_{w}$ are both primitive permutation groups of type PA. Thus $\Gamma$ is a $G$-locally primitive graph of valency $\left|T: C_{T}(\sigma)\right|^{2}$.

Now if we let $\bar{G}=\left(T^{2} \times T^{2}\right):\left(S_{2}\right.$ wr $\left.S_{2}\right)$, where $S_{2}$ wr $S_{2}$ preserves the partition $\{\{1,3\},\{2,4\}\}$, we see that $\bar{G} \leqslant \operatorname{Aut}(\Gamma)$. Also $\bar{G}$ acts quasiprimitively of type CD on both $\Delta_{1}$ and $\Delta_{2}$. Furthermore, $\Gamma$ is $\bar{G}$-locally primitive.

\section{QUASIPRIMITIVE ON ONLY ONE ORBIT}

In this section we prove Theorem 1.3. We begin with the following lemma.

Lemma 7.1. Let $\Gamma$ be a $G$-edge transitive connected graph such that $G$ acts faithfully on its two orbits $\Delta_{1}$ and $\Delta_{2}$ on vertices. Suppose that $G$ has a nontrivial normal subgroup $N$ such that $N_{v}^{\Gamma(v)}=1$ for all $v \in \Delta_{1}$. If there exists $w \in \Delta_{2}$ such that $N_{w}^{\Gamma(w)}=1$, then $N$ acts semiregularly on $V \Gamma$.

Proof. As $G$ is transitive on $\Delta_{2}, N_{w}^{\Gamma(w)}=1$ for all $w \in \Delta_{2}$. The connectivity of $\Gamma$ then implies that $N_{v}$ fixes every element of $\Delta_{1}$ and $\Delta_{2}$. Then since $G$ acts faithfully on $\Delta_{1}, N_{v}=1$ and so $N$ acts semiregularly on $\Delta_{1}$. Also for $w \sim v$, the subgroup $N_{w}$ is contained in $N_{v}=1$. Hence $N_{w}=1$ and so $N$ acts semiregularly on $\Delta_{2}$.

We can now prove Theorem 1.3 .

Proof. Let $\Gamma$ be a locally $(G, s)$-arc transitive connected graph with $s \geq 2$ such that $G$ acts faithfully on its two orbits $\Delta_{1}$ and $\Delta_{2}$ but quasiprimitively on only $\Delta_{1}$. Suppose first that $G$ is quasiprimitive of type HC, SD or CD. Let $X=\operatorname{soc}(G)$. Then for $v \in \Delta_{1}, X_{v}$ is a subdirect subgroup of $X$, so $X_{v} \cong T^{r}$ for some $r \geq 1$. Suppose that $X_{v}^{\Gamma(v)}=1$. Then as $X$ does not act regularly on $\Delta_{1}$, Lemma 7.1 implies that $X_{w}^{\Gamma(w)} \neq 1$ for all $w \in \Delta_{2}$. Now let $w \in \Gamma(v)$ such that $X_{w}$ moves $v$, so $X_{w} \neq X_{v}$. Since $X_{v}^{\Gamma(v)}=1$ it follows that $X_{v}<X_{w}$. Then as $X_{v}$ is a subdirect 
subgroup of $X$, so is $X_{w}$. Thus $X_{w} \cong T^{l}$ for some $l>r$. Furthermore, since $X_{w}^{\Gamma(w)}$ is a nontrivial normal subgroup of the 2-transitive group $G_{w}^{\Gamma(w)}$, a theorem of Burnside [3, Theorem 4.3] implies that $X_{w}^{\Gamma(w)}=T$ and is a primitive group. Thus $X_{w} \cong T^{r+1}$ and $\left(X_{w}\right)_{v}=X_{v} \cong T^{r}$. Since the kernel of $X_{w}$ on $\Gamma(w)$ is contained in $\left(X_{w}\right)_{v}$, it follows that $\left(X_{w}\right)_{v}$ is equal to the kernel, that is, $X_{w}^{\Gamma(w)}$ is regular. This contradicts $X_{w}^{\Gamma(w)}$ being primitive, and we deduce that $X_{v}^{\Gamma(v)} \neq 1$.

Now $X$ is transitive on $\Delta_{1}$, since $G$ is quasiprimitive on $\Delta_{1}$. By Lemma [5.5 and the fact that $X_{v}^{\Gamma(v)} \neq 1$, it follows that $X$ must also be transitive on $\Delta_{2}$. Since $G$ is not quasiprimitive on $\Delta_{2}$ it follows that $X=\operatorname{soc}(G)$ is not a minimal normal subgroup of $G$ and hence $G$ has type HC. Since $X_{v} \cong T^{r}$ we have $X \cong T^{2 r}$ and $r \geq 2$. We have seen that $X_{v}^{\Gamma(v)}$ is a nontrivial normal subgroup of the 2-transitive group $G_{v}^{\Gamma(v)}$. Thus our favourite theorem of Burnside implies that $X_{v}^{\Gamma(v)} \cong T$. However, $X_{v}$ is a minimal normal subgroup of $G_{v}$ and so since it acts nontrivially on $\Gamma(v)$, it acts faithfully on $\Gamma(v)$. Thus $X_{v}^{\Gamma(v)} \cong T^{r}$ which is a contradiction since $r \geq 2$. Thus $G$ is of type HA, HS, AS, PA or TW. We saw in Section 4 that examples of locally $s$-arc transitive graphs for the five types HA, HS, AS, PA and TW do occur. This completes the proof of Theorem 1.3 .

Although there are no locally $(G, 2)$-arc transitive graphs satisfying the conditions of Theorem 1.3 with $G$ quasiprimitive on $\Delta_{1}$ of type HC, SD or CD, there are locally primitive graphs of these types as the following examples demonstrate.

Example 7.2. (1) (HC stars of valency $\left\{n^{2},\left|A_{n-1}\right|^{2}\right\}$ ) Let $\Gamma$ be as in the first example of Example 6.6 and let $G=T^{4}: S_{2}$ where $T=A_{n}$ and $S_{2}$ is generated by the element $(1,2)(3,4)$. Then $\Gamma$ is $G$-locally primitive such that the action of $G$ on $\Delta_{1}=\left[G: G_{v}\right]$ is primitive of type HC. Let $N=T \times T \times 1 \times 1 \triangleleft G$. Then $N$ acts regularly on $\Delta_{1}$ but has $n^{2}$ orbits on $\Delta_{2}$. Thus $\Gamma_{N}$ is the star $K_{1, n^{2}}$.

(2) (Type SD of valency $\{3,|T|\})$ Let $G=T^{3}: S_{3}$ for some nonabelian simple group $T$ and consider the subgroups

$$
G_{v}=\{(t, t, t): t \in T\} \times S_{3}
$$

and

$$
G_{w}=\{(s, t, t): s, t \in T\}:\langle(2,3)\rangle .
$$

Then the graph $\Gamma=\operatorname{Cos}\left(G, G_{v}, G_{w}\right)$ is connected, and $G$ acts primitively of type $\mathrm{SD}$ on $\Delta_{1}=\left[G: G_{v}\right]$. Now

$$
G_{v} \cap G_{w}=\{(t, t, t): t \in T\} \times\langle(2,3)\rangle
$$

which is a maximal subgroup of both $G_{v}$ and $G_{w}$. Thus $\Gamma$ is $G$-locally primitive and biregular of valency $\left\{n^{2},\left|A_{n-1}\right|^{2}\right\}$. The group $G$ does not act quasiprimitively on $\Delta_{2}$, as the normal subgroup $N=T^{3}$ has three orbits. Thus $\Gamma_{N}$ is the star $K_{1,3}$.

(3) (Type CD of valency $\left.\left\{9,|T|^{2}\right\}\right)$ Let $G=T^{6}:\left(S_{3}\right.$ wr $\left.S_{2}\right)$ for some nonabelian simple group $T$. Let

$$
G_{v}=\{(t, t, t, s, s, s): t, s \in T\}:\left(S_{3} \mathrm{wr} S_{2}\right)
$$

and

$$
G_{w}=\left[\left(T \times\left(\{(t, t): t \in T\} \times S_{2}\right)\right) \times\left(T \times\left(\{(t, t): t \in T\} \times S_{2}\right)\right)\right]: S_{2} .
$$


Then the graph $\Gamma=\operatorname{Cos}\left(G, G_{v}, G_{w}\right)$ is connected and $G$ acts primitively of type CD on $\Delta_{1}=\left[G: G_{v}\right]$. Now

$$
G_{v} \cap G_{w}=\{(t, t, t, s, s, s): t, s \in T\}:\left((\langle(2,3)\rangle \times\langle(2,3)\rangle): S_{2}\right)
$$

which is a maximal subgroup of $G_{v}$ and $G_{w}$. Thus $\Gamma$ is $G$-locally primitive and biregular of valency $\left\{9,|T|^{2}\right\}$. Now $G$ does not act quasiprimitively on $\Delta_{2}$ as $N$ has nine orbits, so $\Gamma_{N}$ is the star $K_{1,9}$.

\section{Some open PRoblems}

The work of this paper directs our attention to studying the "basic" locally $(G, s)$-arc transitive graphs with $s \geq 2$, that is, upon the following two problems.

Problem 8.1. Further analyse the structure and properties of locally $(G, s)$-arc transitive graphs, for which $G$ acts faithfully and quasiprimitively on both orbits of $G$ on vertices.

Problem 8.2. Further analyse the structure and properties of locally $(G, s)$-arc transitive graphs, for which $G$ acts faithfully on both orbits $\Delta_{1}$ and $\Delta_{2}$ on vertices, but $G$ acts quasiprimitively only on $\Delta_{1}$.

For some of the quasiprimitive types given in the first problem it would seem feasible to determine, or at least provide a nice characterisation of, such graphs. The $\{\mathrm{SD}, \mathrm{PA}\}$ case is currently being considered by the authors. In the case where $G$ acts quasiprimitively of type PA on both orbits, the only known examples are the standard double covers of $(G, s)$-arc transitive graphs as given in Lemma 3.3 It would be interesting to find examples of PA type which did not arise in this way.

The second problem has been looked at in [12] where a nice characterisation of such graphs has been found which enables the vertices of $\Delta_{2}$ to be represented by certain subsets of $\Delta_{1}$. In the case where $G$ is quasiprimitive of type HA, HS or TW on $\Delta_{1}$, we can identify $\Delta_{1}$ with a minimal normal subgroup $N$ of $G$ and the problem is reduced to finding certain collections of $p$-subgroups of $N$.

The paper 10 determines all locally $(G, s)$-arc transitive graphs where $G$ is a Ree simple group acting quasiprimitively on both of its orbits. Three new infinite families of examples are constructed.

Problem 8.3. Classify all locally $(G, s)$-arc transitive graphs for other families of small rank Lie type groups $G$.

Placing further restrictions on $s$ decreases the possibilities for the "basic" graphs. For example, Lemma 5.6 implies that when $s \geq 4$, we need to study only the case where $G$ acts quasiprimitively on both orbits. When $G$ is quasiprimitive of type HA on both $G$-orbits, it was shown in [15, Lemma 3.2] that $\Gamma$ has an automorphism $\tau$ of order two which interchanges $\Delta_{1}$ and $\Delta_{2}$. Then $\langle N, \tau\rangle$ is a regular normal subgroup of $\bar{G}=\langle G, \tau\rangle$. Hence by [18, Proposition 3.2], $\Gamma$ is not $(\bar{G}, s)$-arc transitive for $s \geq 4$ and so $\Gamma$ is not locally $(G, s)$-arc transitive for $s \geq 4$.

Question 8.4. Given a locally $(G, s)$-arc transitive graph with $s \geq 3$ and $G$ acting faithfully on both $\Delta_{1}$ and $\Delta_{2}$ and quasiprimitively on at least one, what are the possible quasiprimitive types for $G$ ? Similarly, find the possible quasiprimitive types for $G$ when $s \geq 4$.

Given a locally $(G, s)$-arc transitive graph $\Gamma$ it would be interesting to determine the full automorphism group. This leads us to the following question. 
Question 8.5. When is a locally $(G, s)$-arc transitive graph Aut $(\Gamma)$-vertex transitive?

Clearly $\Gamma$ has to be regular, and so determining when $\Gamma$ is regular would be a good first step. In Section 3 we discussed some sufficient conditions when all vertex stabilisers were conjugate in $G$. A regular graph which is edge transitive but not vertex transitive is called semisymmetric. Such graphs have been studied extensively; see for example 9], 11] and [15. The smallest number of vertices for a semisymmetric graph is 80 , see 8], and there are two such graphs, one of which is a generalised 4 -gon and hence locally 5 -arc transitive.

In 31, Weiss conjectured that for a $G$-vertex transitive, $G$-locally primitive graph of valency $k$, the order $\left|G_{v}\right|$ of a vertex stabiliser is bounded above by a function of $k$. For the nonbipartite case, it was proved in [4] that the conjecture holds if and only if it holds in the case where $G$ is an almost simple group. In 20] it was conjectured that the same result holds for the more general class of $G$-vertex transitive $G$-locally quasiprimitive graphs. It was mentioned in 31] that the case where $\Gamma$ is not $G$-vertex transitive should also be investigated. As currently stated the conjecture does not hold even for locally $3-\operatorname{arc}$ transitive graphs. Vertices in a $G$-locally primitive graph have valency $k_{1}$ if they lie in $\Delta_{1}$ and $k_{2}$ if they lie in $\Delta_{2}$ with $k_{1}$ and $k_{2}$ not necessarily equal. There are infinite families of graphs for which $k_{2}$ is constant while $k_{1}$, and hence $\left|G_{v}\right|$ for some vertex $v$ of valency $k_{2}$, are unbounded. For example, the graphs in Example 4.2 constructed from the points and lines of $\operatorname{AG}(d, q)$ for a fixed $q$ and unbounded $d$. With this in mind we make the following conjecture.

Conjecture 8.6. If $\Gamma$ is a connected $G$-locally primitive graph such that $G$ has two orbits on vertices and $\Gamma$ is biregular of valency $\left\{k_{1}, k_{2}\right\}$, then the order $\left|G_{e}\right|$ of an edge stabiliser is bounded by a function of $\max \left\{k_{1}, k_{2}\right\}$.

\section{REFERENCES}

[1] Robert W. Baddeley, Two-arc transitive graphs and twisted wreath products, J. Algebraic Combin. 2 (1993), 215-237. MR 94h:05037

[2] I. Z. Bouwer and D. Ž. Djoković, On regular graphs. III, J. Combinatorial Theory (B) 14 (1973), 268-277. MR 47:4858

[3] Peter J. Cameron, Permutation groups, London Mathematical Society Student Texts, no. 45, Cambridge University Press, Cambridge, 1999. MR 2001c:20008

[4] Marston D. Conder, Cai Heng Li, and Cheryl E. Praeger, On the Weiss conjecture for finite locally primitive graphs, Proc. Edinburgh Math. Soc. 43 (2000), 129-138. MR 2001e:05054

[5] J. H. Conway, R. T. Curtis, S. P. Norton, R. A. Parker, and R. A. Wilson, Atlas of finite groups, Clarendon Press, Oxford, 1985. MR 88g:20025

[6] A. Delgado, D. Goldschmidt, and B. Stellmacher, Groups and graphs: new results and methods, Birkhäuser Verlag, Basel, 1985. MR 88a:05076

[7] P. Dembowski, Finite geometries, Springer-Verlag, Berlin, 1968. MR 38:1597

[8] Shaofei Du and Dragan Marušič, Biprimitive graphs of smallest order, J. Algebraic Combin. 9 (1999), 151-156. MR 2000b:05069

[9] Shaofei Du and Mingyao Xu, A classification of semisymmetric graphs of order $2 p q$, Comm. Algebra 28 (2000), 2685-2715. MR 2001g:05059

[10] Xin Gui Fang, Cai Heng Li, and Cheryl E. Praeger, On locally two-arc transitive graphs admitting a Ree simple group, in preparation.

[11] Jon Folkman, Regular line-symmetric graphs, J. Combinatorial Theory 3 (1967), 215-232. MR 37:97

[12] Michael Giudici, Cai Heng Li, and Cheryl E. Praeger, Characterising finite locally s-arc transitive graphs with a star normal quotient, in preparation. 
[13] _ Some locally 3-arc transitive graphs constructed from triality, submitted.

[14] David M. Goldschmidt, Automorphisms of trivalent graphs, Ann. of Math. (2) 111 (1980), 377-406. MR 82a:05052

[15] A. A. Ivanov and M. E. Iofinova, Biprimitive cubic graphs, Investigations in the algebraic theory of combinatorial objects, Kluwer, 1993, pp. 459-472.

[16] A. A. Ivanov and Cheryl E. Praeger, On finite affine 2-arc transitive graphs, Europ. J. Combinatorics 14 (1993), 421-444. MR 94k:05089

[17] Cai Heng Li, On finite moufang graphs, in preparation.

[18] Finite s-arc transitive graphs of prime-power order, Bull. London Math. Soc. 33 (2001), 129-137. MR 2002d:05064

[19] - The finite vertex-primitive and vertex-biprimitive s-transitive graphs for $s \geq 4$, Trans. Amer. Math. Soc. 353 (2001), 3511-3529. MR 2002c:05084

[20] Cai Heng Li, Cheryl E. Praeger, Akshay Venkatesh, and Sanming Zhou, Finite locallyquasiprimitive graphs, Discrete Math. 246 (2002), 197-218. MR 2003c:05110

[21] Martin W. Liebeck, Cheryl E. Praeger, and Jan Saxl, On the O'Nan-Scott Theorem for finite primitive permutation groups, J. Austral. Math. Soc. (A) 44 (1988), 389-396. MR 89a:20002

[22] Cheryl E. Praeger, On a reduction theorem for finite, bipartite 2-arc-transitive graphs, Australas J. Comb. 7 (1993), 21-36. MR 93m:05091

[23] Cheryl E. Praeger, An O'Nan-Scott theorem for finite quasiprimitive permutation groups and an application to 2-arc transitive graphs, J. London Math. Soc. (2) 47 (1993), 227-239. MR 94f:05068

[24] Cheryl E. Praeger, Finite quasiprimitive graphs, Surveys in combinatorics, 1997. Proceedings of the 16th British combinatorial conference, London, UK, July 1997 (R. A. Bailey, ed.), Lond. Math. Soc. Lect. Note Ser., no. 241, Cambridge University Press, 1997, pp. 65-85. MR 99b:05076

[25] Gert Sabidussi, Vertex-transitive graphs, Monatsh. Math. 68 (1964), 426-438. MR 31:91

[26] D. H. Smith, Primitive and imprimitive graphs, Quart. J. Math. Oxford (2) 22 (1971), 551557. MR 48:5926

[27] Bernd Stellmacher, Locally s-transitive graphs, unpublished.

[28] Michio Suzuki, Group theory. I, Springer-Verlag, Berlin, 1982. MR 82k:20001c

[29] W. T. Tutte, A family of cubical graphs, Proc. Cambridge Philos. Soc. 43 (1947), $459-474$. $\operatorname{MR~9:97g~}$

[30] - On the symmetry of cubic graphs, Canad. J. Math. 11 (1959), 621-624. MR 22:679

[31] R. Weiss, s-transitive graphs, Algebraic methods in graph theory, Vol. I, II (Szeged, 1978), North-Holland, Amsterdam, 1981, pp. 827-847. MR 83b:05071

[32] Richard Weiss, The nonexistence of 8-transitive graphs, Combinatorica 1 (1981), 309-311. MR 84f:05050

[33] Richard Weiss, Generalized polygons and s-transitive graphs, Finite geometries, buildings, and related topics (Pingree Park, CO, 1988), Oxford Univ. Press, New York, 1990, pp. 95-103. MR 91j:51011

School of Mathematics and Statistics, The University of Western Australia, 35 Stirling Highway, Crawley, Western Australia 6009, Australia

E-mail address: giudici@maths.uwa.edu.au

School of Mathematics and Statistics, The University of Western Australia, 35 Stirling Highway, Crawley, Western Australia 6009, Australia

E-mail address: li@maths.uwa.edu.au

School of Mathematics and Statistics, The University of Western Australia, 35

Stirling Highway, Crawley, Western Australia 6009, Australia

E-mail address: praeger@maths.uwa.edu.au 